\title{
25 Research Square \\ Selection and impact of leading edge on boundary layer transition
}

\author{
Dinesh Devraj Bhatia ( $\nabla$ ddbhatia@gmail.com ) \\ University of Nottingham Ningbo China https://orcid.org/0000-0002-9803-377X \\ Guangjun Yang \\ Northwestern Polytechnical University School of Aeronautics \\ Guangning Li \\ Northwestern Polytechnical University School of Astronautics \\ Jian Wang \\ Kingston University
}

\section{Research}

Keywords: Boundary Layer Transition, Leading Edge, Aspect Ratio, Curvature

Posted Date: May 26th, 2020

DOl: https://doi.org/10.21203/rs.3.rs-29913/v1

License: (9) This work is licensed under a Creative Commons Attribution 4.0 International License. Read Full License 


\title{
Selection and impact of leading edge on boundary layer transition
}

\author{
D. Bhatia ${ }^{1 \dagger}$, G.Yang $^{2}, \mathrm{G.Li}^{2}$, and J. Wang ${ }^{3}$ \\ ${ }^{1}$ School of Aerospace Engineering, The University of Nottingham Ningbo China, Ningbo, China \\ 315000 \\ 2 School of Aeronautics, Northwestern Polytechnical University, Xi'an, China 710072 \\ ${ }^{3}$ Faculty of Science, Engineering and Computing, Kingston University London, London, United \\ Kingdom SW15 3DW \\ †Corresponding Author Email: ddbhatia@gmail.com
}

\begin{abstract}
The choice of leading edge aspect ratio (AR) plays a crucial role when planning boundary layer experimental wind tunnel tests on a flat plate. Poor selection of the leading-edge profile hampers the effectiveness of the experiment and increases testing costs associated with interchanging of leading edges to attain accurate results. Thus, the appropriate selection of the leading edge is a very crucial part of the wind tunnel experimental process. The authors, in this paper, argue that the curvature of the leading edge and thus the AR are of paramount importance to attain accurate results from wind tunnel testing. In this paper, the authors have tested 7 different elliptical leading edges and compared their performance with an ideal leading edge with zero thickness. Experimental and computational has been presented for leading edges ranging from AR6 to AR20. Results were evaluated for boundary layer transition onset location and it was found that AR20 has the least influence on the flow structure when compared the ideal leading edge. A study of the flow structure at the stagnation point indicates an increase in adverse pressure gradient with an increase in the AR but also a decrease in the size of the stagnation region. The presence of a higher AR leading edge reduces the turbulent spot production rate which is one of the primary causes of boundary layer transition. The authors have presented a correlation which makes it easier for aerodynamicists to quantify the impact of the leading-edge AR on transition. A case is also presented to compare the relative performance of a wedge and the higher AR leading edge which gives potential researchers the choice between an elliptical or a wedge-shaped leading edge.
\end{abstract}

Keywords: Boundary Layer Transition, Leading Edge, Aspect Ratio, Curvature

\section{INTRODUCTION}

Boundary layer transition is influenced by numerous factors including changes in the surface curvature, turbulence intensity, surface roughness and the velocity of the flow. In a controlled environment wherein the turbulence intensity, velocity of the flow and the flat plate are unchanged, a change in the aspect ratio of the leading edge will be the primary cause for changes in the transition onset location. The primary factor, in this case, is the change in the surface curvature. Görtler (1941) through his series of experiments found that the presence of a convex curved surface tends to stabilize the boundary layer while a concave surface tends 
to destabilize the boundary layer. Görtler found that a laminar boundary layer on a concave surface becomes unstable as a result of the centrifugal force and three-dimensional disturbances whereas boundary layers on convex surfaces are free from centrifugal forces. Consequently, the effects of concave surface geometry have an impact on the transition onset location by up to $56 \%$ (Schultz \& Volino, 2003). Thus, it can be said that the curvature (whether convex or concave) of the leading edge can influence the location of boundary layer transition. In the case of convex surfaces (as is the case with the elliptical leading edges in the present case) the impact of surface curvature has been well documented in experimental studies such as the one conducted by Mayle (1991). According to Mayle, a change in the surface curvature results in a change in the turbulent spot propagation rate $\widehat{n} \sigma$. Larger adverse pressure gradient would lead to greater spot propagation rates. These spot propagation rates have a direct impact on boundary layer transition. Changes in the leading edge Aspect Ratio also lead to changes in the pressure distribution along the leading edge (Kendall, 1991). In coherence with the research conducted by Mayle and Kendall, Klingmann et al. (1993) are also of the opinion that turbulent spot propagation is directly impacted by changes in the AR due to changes in the surface curvature. Higher adverse pressure gradient along the leading edge would result in greater spot propagation which in turn would impact the transition onset location.

During the study of boundary layers over flat plates, it was found that the leading edge is a particularly sensitive region as this is where the disturbance first enters the boundary layer (Hanson, et al., 2012). The turbulent spot propagation rate can, thus, be thought of as an indicator of the magnitude of disturbance that enters the boundary layer due to the presence of the leading edge aspect ratio. This can further be corroborated by the conclusions proposed by Saric et al. (1999) which indicate that, the receptivity of the boundary layer to freestream disturbances greatly depends on the leading edge curvature, discontinuities in the surface curvature as well as surface inhomogeneities. Experimental investigation to understand the effect of leading edge bluntness on boundary layer development has shown that an increase in the Aspect Ratio (AR) of the leading edge leads to a decrease in the amplitude of TollmienSchlichting (TS) waves which are the primary manifestation of disturbances in the boundary layer (Kendall, 1991). Saric et al. isolated the leading edge to quantify the effect of the leading edge on the initial amplitude of the TS waves. Some of the key conclusions from elliptical leading edge tests by Saric et al. are as follows (Saric, et al., 1999):

a) TS wave amplitude decreases when the elliptical nose is sharpened i.e. AR increases

b) The smoothening of the discontinuity at the ellipse/flat plate results in a reduction in the amplitude of TS waves by about $50 \%$

c) The disturbance which is responsible for the generation of the TS waves originates from the location of the maximum adverse pressure point and depends on the magnitude of the adverse pressure

The consequence of an increase in the Aspect ratio is well documented in the previous paragraphs and indicates that an increase in the Aspect Ratio would lead to a delay in the transition. However, according to Nagarajan et al. (2007), a change in the Aspect ratio does not affect the transition onset location in a predictable manner and the transition onset location in these situations varies greatly with each change in AR. 
There exists a lack of database which clearly highlights the appropriate choice of leading edge for computational, experimental or numerical research. While all previously documented literature has discussed in detail about the impact of surface curvature on boundary layer transition, very little information about integrated viewpoints is available of the impact of the different aspect ratio leading edge and their influence on the boundary layer and flow transition under uniform flow conditions. This research endeavour lays emphasis on the ascertaining and quantifying the impact of the change in curvature on boundary layer transition. The generation of a database of different aspect ratio leading edges and their impact on boundary layer transition is the other important aim of this paper.

The ERCOFTAC T3A, T3B, and T3C experiments are used as benchmark cases in a number of experimental and computational research cases with zero pressure gradients (Langtry, et al., 2004). The T3 test cases make use of a semi-circular leading edge. Lin et al. (2012) found out that while using the T3 test cases for simulations, little or no information is available on the impact of changes in the gradient of elliptical leading edges on boundary layer and transition measurements. Thus, there is a dearth of data available for the optimal leading edge to be used for simulations and experiments. This lack of data is extremely crucial because each small change in leading edge geometry can influence transition. It is important to note that for an elliptical leading edge, there might be no separation bubble as in the case of a blunt leading edge. Changes of leading edge aspect ratio will lead the increase of elliptical curvature at the tip and curvature decrease at other places. The impact of changes in the curvature on the stagnation point, on rest part of the elliptic curve and connection of the elliptic and flat plate as well as the correlation between the curvature and the pressure gradient, have not been studied in isolation. The changes in the flow structure based on the change in the geometry need to be investigated in detail. The presence of a sharp leading edge theoretically should have the best aerodynamic performance. Therefore, an analysis needs to be done to find out the impact of increasing AR on transition onset and its comparison to a theoretical leading edge with $A R^{\infty}$. Research has also been conducted to identify a "modified super ellipse" that could be used for a number of computational and practical test cases. This super ellipse could possibly eliminate the leading edge/flat plate juncture discontinuity, by smoothing out the juncture discontinuity by using a polynomial function (Hanson, et al., 2012). One of the key results from the experimental and computation tests was that the presence of a "modified super ellipse" can lead to minimizing the region of non-zero pressure gradients which reduces the receptivity of the boundary layer to disturbances. However, while, this super ellipse has been tested in a couple of test cases its implementation in a number of different test scenarios could be cumbersome when a number of different aspect ratio leading edges are to be tested. Thus, in such cases, it is necessary to find an optimum leading edge that would serve the purpose for both experimental as well as computational test scenarios.

The authors have conducted a number of experiments as well as simulations by varying the aspect ratios of the leading edge and analysing the effects of these changes on boundary layer transition. The concept and the detailed setup for the present research work and presented in the next section. All experiments and simulations have been conducted under a uniform set of test conditions and a number of different test cases have been simulated keeping all parameters exactly the same. The present study addresses the significance of an elliptical leading edge on boundary layer transition and sheds some light on the appropriate leading edge that could possibly be used by researchers depending on the accuracy sought by researchers in individual test cases by means of providing the comparison of different practical aspect ratios with a theoretical leading edge with $A R \infty$. The notion that an elliptical leading 
edge is the most appropriate geometric configuration has also been challenged with the use of a wedge-shaped leading edge.

\section{CONCEPT AND METHODOLOGY}

\subsection{Concept}

To study the impact of changes in AR of elliptical leading edges, the authors have focused on the impact of changes in geometry of the ellipse and identified four key areas mainly the stagnation region, the change of curvature along the length of the leading edge, the pressure gradient and its changes along the length of the leading edge, and the turbulent spot propagation rate due to a change in the aspect ratio.

To compare different leading edges, an ideal leading edge with zero thickness and infinite length has been considered i.e. $A R^{\infty}$. Comparison to $A R^{\infty}$ would indicate the receptivity of the boundary layer to disturbances due to the presence of a change in geometry. In addition, comparison to $A R \infty$ will provide the required information to select a practical and optimum leading edge for future wind tunnel tests as well as simulations.

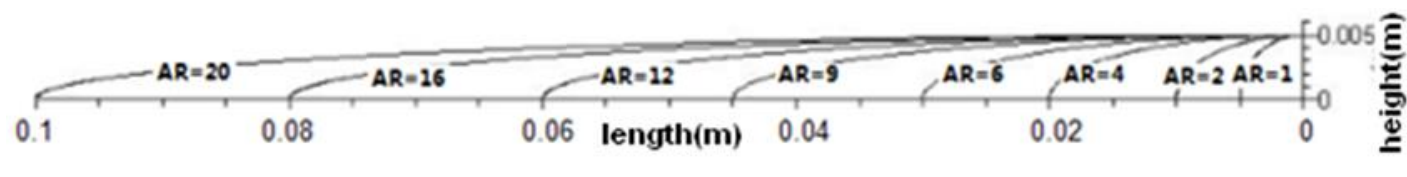

Figure 1 Individual profiles for different AR leading edges

\subsubsection{Curvature $\mathrm{k}$}

When elliptical leading edges are used, as the AR changes, the curvature of the leading edge also changes. The nose of the leading edge as well as regions wherein curvature discontinuity/sharp changes in curvature exist (e.g. the joint of the elliptical leading edge to the flat plate section) tend to provide regions of high receptivity (Hanson, et al., 2012). The rate of change of curvature along the length of the leading edge can give insights into the flow of the fluid over leading edge. At the start of the leading edge, the curvature for each different AR leading edge is different. A study of the change in the curvature of each individual leading edge is a possible indicator as to the flow development on the leading edge. The pressure gradient will also change with a change in curvature. When the surface is convex as is the case for all elliptical leading edges in the present research, the turbulent spot propagation rates would be directly correlated to the curvature. The spot propagation rate can be used as an indicator of the impact of the leading edge on boundary layer transition. The curvature change can also be linked to a change in the pressure gradient. The aerodynamic performance of test specimen depends on the impact of the leading edge and a change in the curvature would alter the pressure gradient. The curvature changes at different points on each leading edge have been studied numerically and theoretically. A comparison of the curvature change at specific points on each leading edge will highlight the impact of changes in the aspect ratio on the fluid flow. Thus, it can be said that the curvature will have a direct impact on the stagnation region, the pressure gradient as well as the turbulent spot propagation rate.

As all leading edges are elliptical in nature, the fundamental equations for calculating the radius $R$, curvature $K$ as well as gradient for each individual leading edge are derived from the fundamental equation of the ellipse (Weisstein, 2015):

$\frac{x^{2}}{a^{2}}+\frac{y^{2}}{b^{2}}=1$ 
Where,

$x, y: \mathrm{X}$ and $\mathrm{Y}$ co-ordinates for the ellipse respectively

$a$ : Major Radius for the ellipse

$b:$ Minor radius for the ellipse

The curvature $\mathrm{k}$ and the radius $\mathrm{R}$ are calculated using the equations (Weisstein, 2015):

$\kappa=\frac{\frac{d^{2} y}{d x^{2}}}{\left[1+\left(\frac{d y}{d x}\right)^{2}\right]^{3 / 2}}$

Where,

$\frac{d y}{d x}, \frac{d^{2} y}{d x^{2}}:$ First and second order differential equations for Eq. (1)

And,

$R=\frac{1}{\kappa}$

\subsubsection{Stagnation Region}

The stagnation region is the region of low-velocity fluid just fore of the nose of the leading edge $(x / L=0)$. The fluid encounters the leading edge and the presence of the elliptical leading edge results in this region of low velocity, high-pressure region. The radius of the leading edge (hence curvature) at the nose plays a significant role in determining the structure of the stagnation region. The size of the stagnation region could impact the flow later on along the leading edge and could be the first region where the disturbance might start to enter the boundary layer. Figure 1 shows the individual profile of each leading edge and indicates that the radius of the curve at the start of the leading edge reduces with an increase in AR.

\subsubsection{Pressure Gradient}

One effect of the change in the curvature is the alteration of the pressure gradient. In addition, destabilization of the boundary layer takes place due to presence of adverse pressure gradients and other factors. The presence of adverse pressure gradients increases the rate of the growth of the disturbance and enhances the receptivity of the boundary layer to disturbances (Zaki \& Durbin, 2006). The presence of a pressure gradient leads to an acceleration or deceleration of flow around the flat plate. This acceleration or deceleration (change in pressure gradient) affects the transition to a certain extent. Negative acceleration takes place at adverse pressure gradients (Mayle, 1991). Research conducted by Schraub \& Kline (1965) further exemplify that the increase in the adverse pressure gradient results in a much larger spot propagation rate and hence a quicker transition and vice-versa. The pressure gradients, in this case, are directly linked to changes in the curvature of different elliptical leading edges. The pressure gradients have been studied at several key points along different leading edges and a direct link can be established between the curvature change and the pressure gradient. The critical point at which the favourable pressure gradient on the leading edge changes to adverse pressure gradient will have a direct impact on the transition onset as all other factors apart from the curvature of the leading edges are unchanged.

\subsubsection{Turbulent Spot Propagation Rate $(\widehat{n} \sigma)$}


The changes in the curvature enhance the receptivity of the boundary layer to disturbances. The spot propagation rate is an indicator of the disturbances in the boundary layer. The turbulent spot propagation rates increase with an increase in adverse pressure gradient as discussed in the preceding section. With all other parameters being kept constant, it can be said that the spot propagation depends solely on the curvature and hence the aspect ratio of the leading edge.

The turbulent spot propagation rate can be calculated using the equation (Schultz \& Volino, 2003):

$\widehat{n} \sigma=\frac{4.6 v^{2} U_{0}}{\left(x_{e}-x_{t}\right)^{2} U_{t}^{3}}$

\subsection{Methodology}

To investigate the proposed issues, wind tunnel experiments were conducted first to see if there are significant effects of curvature change on transition. Then, analytic formulae and methods were applied to study characteristics of the curvature of the ellipse. Then, CFD was used to compute the effects of changes of curvature on flow transition. Finally, efforts were devoted to identify the trends in the data and correlate relevant parameters to provide insight of proposed investigation.

\subsubsection{Experimental Setup}

The present set of experimental test cases were performed in a LTWT (Low Turbulence Wind Tunnel) which was characterised by an extremely low turbulence intensity of $0.018 \%$ in the velocity interval of $5 \mathrm{~ms}^{-1}-75 \mathrm{~ms}^{-1}$. The experimental set-up is very similar to the ones conducted by previous researchers such as Westin et al. (1994) and Klingmann et al. (1993) and uses a flat plate which is undisturbed and has zero pressure gradient. The details of the wind tunnel and its construction are given by Bhatia et al. (2014). The experiments were carried out on a plate that was $2.2 \mathrm{~m}$ long, $0.396 \mathrm{~m}$ wide and $0.012 \mathrm{~m}$ thick. The streamwise direction was denoted as $x$. Similarly, for the wall normal and the spanwise directions, denotions of $y$ and $z$ were used respectively. Velocity measurements in the $x, y \& z$ directions were done by making use of constant temperature hot-wire anemometers. The probe used in the experimentation was a DANTEC 55P11 single probe. The Constant Temperature Anemometry (CTA) equipment has a measurement accuracy of Tu $<0.015 \%$ (based on the ripple voltage) and $\Delta \mathrm{V}<0.3 \%$ (based on the speed calibration). A commercial hot wire anemometry software provided by DANTEC was used to calibrate hot wire probes. The miniCTA software provided by DANTEC offers the option of online calibration and curve fitting (Dantec Dynamics, 2013). 


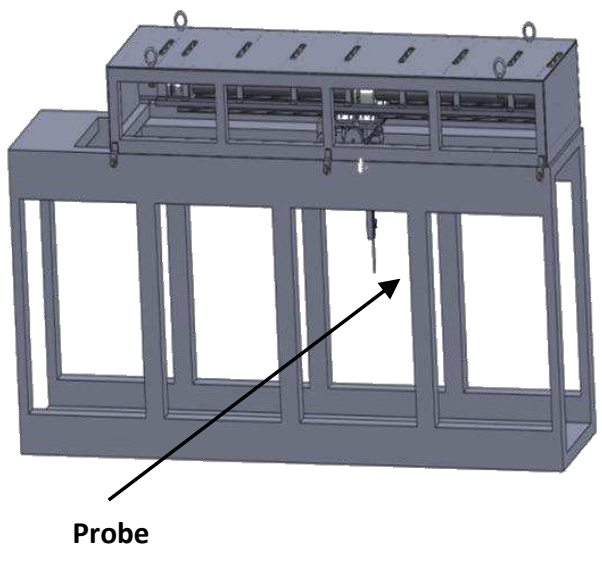

Figure $\mathbf{2}$ Schematics of the test assembly

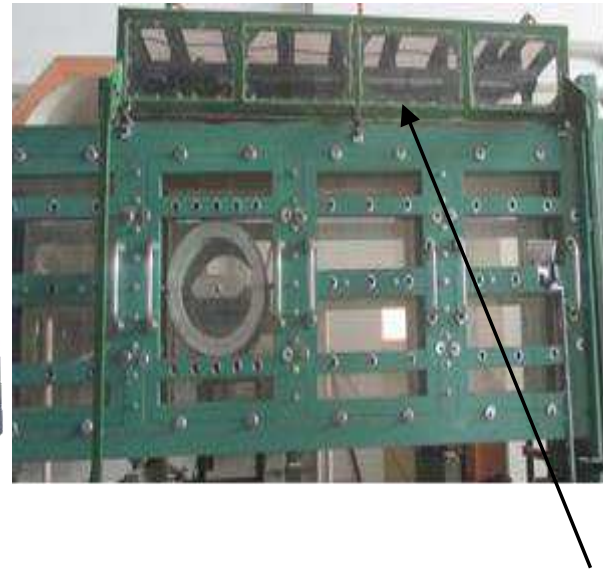

Linear Traverse

Figure 2 depicts the linear traverse probe which is installed above the 2D section of the wind tunnel. The linear traverse probe consists of 5 main elements: An X-axis moving system, a Z axis and a $Y$-axis moving system along with control system used along with a DC motor as a servo system to drive each axis along with a ball screw pair to transmit the motion. The effective stroke of the $X$ axis is $1500 \mathrm{~mm}$ with a minimum moving distance of $1.0 \mathrm{~mm}$ and an accuracy of $0.1 \mathrm{~mm}$. The $Y$-axis stroke is $130 \mathrm{~mm}$ with a minimum moving distance of $0.1 \mathrm{~mm}$ and an accuracy of $0.02 \mathrm{~mm}$. The effective stroke of the $Z$ axis is $200 \mathrm{~mm}$, the minimum moving distance is $0.1 \mathrm{~mm}$ and the accuracy is $0.02 \mathrm{~m}$. To ensure that the data acquired from the wind tunnel is accurate and repeatable, an error analysis was done for the LTWT. The data acquisition equipment in the Low Turbulence Wind Tunnel Laboratory (LTWT) adheres to the following standards:

- National Standard for Flow Field Qualities of High Speed and Low-Speed Wind Tunnel (GJB1179-91). 1991.

- National Standard for Experimental Precision of High Speed and Low-Speed Wind Tunnel (GJB1061-91). 1991.

The test cases (sample models) involved in the experimental work were AR1 (1:1), AR2 (2:1), AR4 (4:1), AR6 (6:1), AR9 (9:1) and AR12 (12:1) although the current paper highlights the results for AR6, AR9, and AR12. The schematics of the test setup including constant temperature anemometry (CTA) and the linear traverse along with the some sample leading edges are shown in Figure 2 and Figure 3. A blockage analysis was done for the wind tunnel setup and it was found that the flow in the wind tunnel was affected by the presence of the linear traverse arm. The blockage effect was found to affect the transition onset location by about 20\% (Bhatia, 2016). The impact of the surface roughness was also quantified and it was found that the surface roughness of the plate can impact the boundary layer transition by up to $9 \%$ (Bhatia, 2016). Thus, it can be assumed that the impact of the blockage and the flat plate surface roughness can impact the boundary layer transition by up to $29 \%$. This will be reflected in the experimental results presented in Section 3.1 


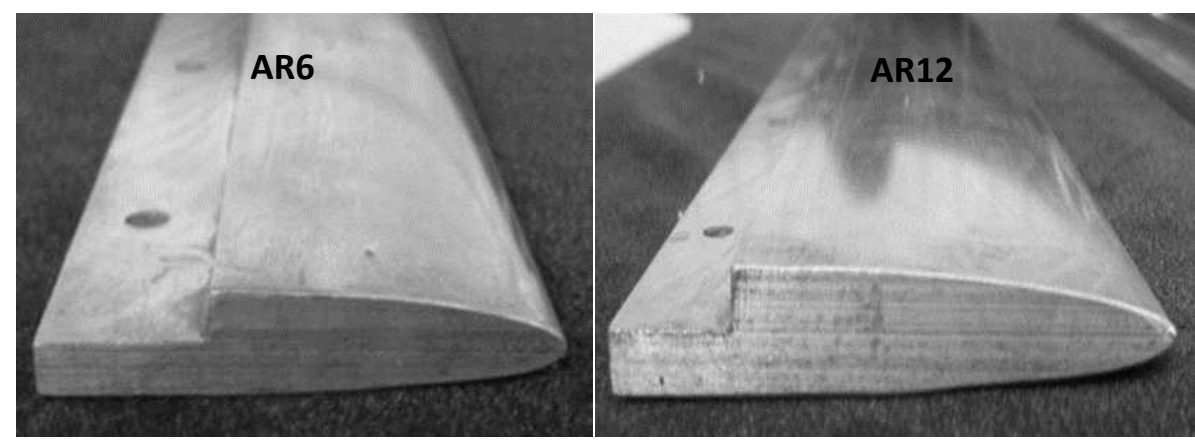

Figure 3 Sample leading edges for the wind tunnel test

\subsubsection{Simulation Setup}

The flat plate geometry used for the simulation is a $2 \mathrm{D} 1.8 \mathrm{~m}$ long and $0.012 \mathrm{~m}$ thick flat plate with AR12 as the default leading edge for all reliability calculations. This plate is identical to the plate used by Lin et al. (2012) in their series of simulations. Consequently, the flat plate is modified for changes in the AR. All other parameters are kept constant. The simulation setup and the test cases in this paper are similar to the experimental work conducted by Bhatia et al (Bhatia, et al., 2014).

The computational domain consisted of the velocity inlet being placed at $0.12 \mathrm{~m}$ from the leading edge of the plate. The height of the domain is kept at $0.147 \mathrm{~m}$ and extends $0.5 \mathrm{~m}$ downstream.

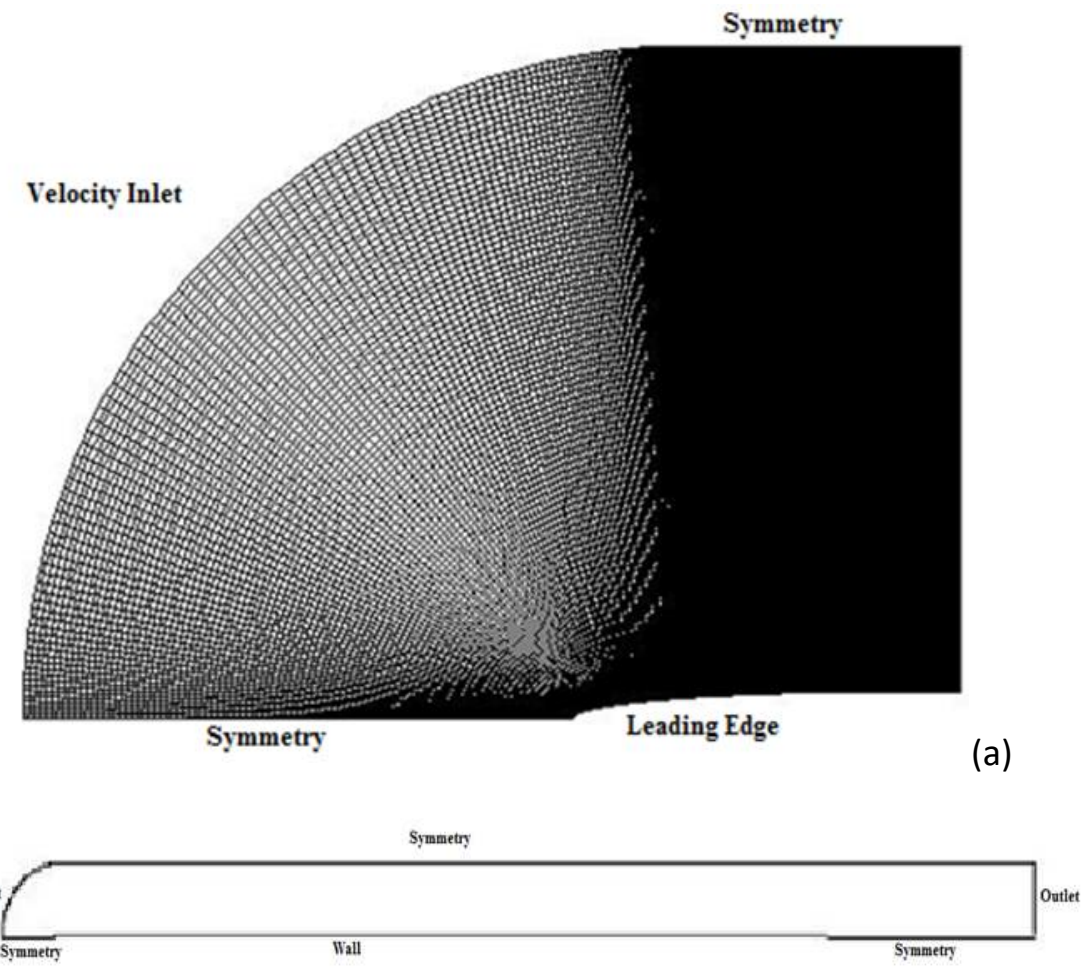

(b)

Figure 4 Mesh Distribution (a) and Boundary conditions (b) along the flat plate

The mesh used for the simulations is a structured multi-block mesh with the mesh being clustered around the leading edge. A maximum $y+$ value of 0.1 is maintained throughout the grid and the wall normal expansion ratio is set to 1.07. A mesh with 400k elements is chosen for the simulation based on grid independence studies and as optimum balance between accuracy and computational processing requirements. A no-slip wall boundary condition was 
used for the flat plate along with a velocity inlet and a pressure outlet. All other planes of the domain are given a symmetry boundary condition.

For the present research, it was decided to incorporate the Transition SST model in FLUENT for a flat plate with different geometric configurations. All model constants for the simulations were kept exactly at their default values.

It was found from preliminary simulations and previous work conducted by Lin et al. (2012) that natural transition was not sensitive to the viscosity ratio between a range of 5-10. It was decided to take the higher value of 10 in this case as the default value for all simulations i.e. $\mu_{t}=10$. The test cases for the simulations are AR9, 12, 16, 20 and $\infty$. All results presented in Section III are compared to $A R \infty$ as the reference value. Benchmarking was run to ensure that the authors work are highly accurate and repeatable. In the present case, benchmarking for the simulations was carried out in accordance with the experimental flat plate tests conducted by Schubauer and Klebanoff (1955) and simulations based on these flat plate tests by (Menter, et al., 2006), (Malan, et al., 2009) and Lin et al. (2012). Figure 5 indicates the skin friction coefficient of fluid flow over a flat plate. The blue line indicates the project simulation results as carried out by the author and the red colour line indicates the benchmark results as carried out by Lin et al. The graph indicates that the curve for $C_{f} v s$. R $e_{x}$ in the author's case is in close accordance with the benchmarking curve. The transition occurs at a very similar value of $2.85 \times 10^{6}$ to that of the experimentally determined value of Reynolds number of $2.8 \times 10^{6}$. A slight discrepancy in the results between the author and Lin et al. can be attributed to the difference in the way the transition model is incorporated in FLUENT as compared to that in Star CCM+. Star CCM+ has an additional boundary layer code incorporated in its model (Malan, et al., 2009).

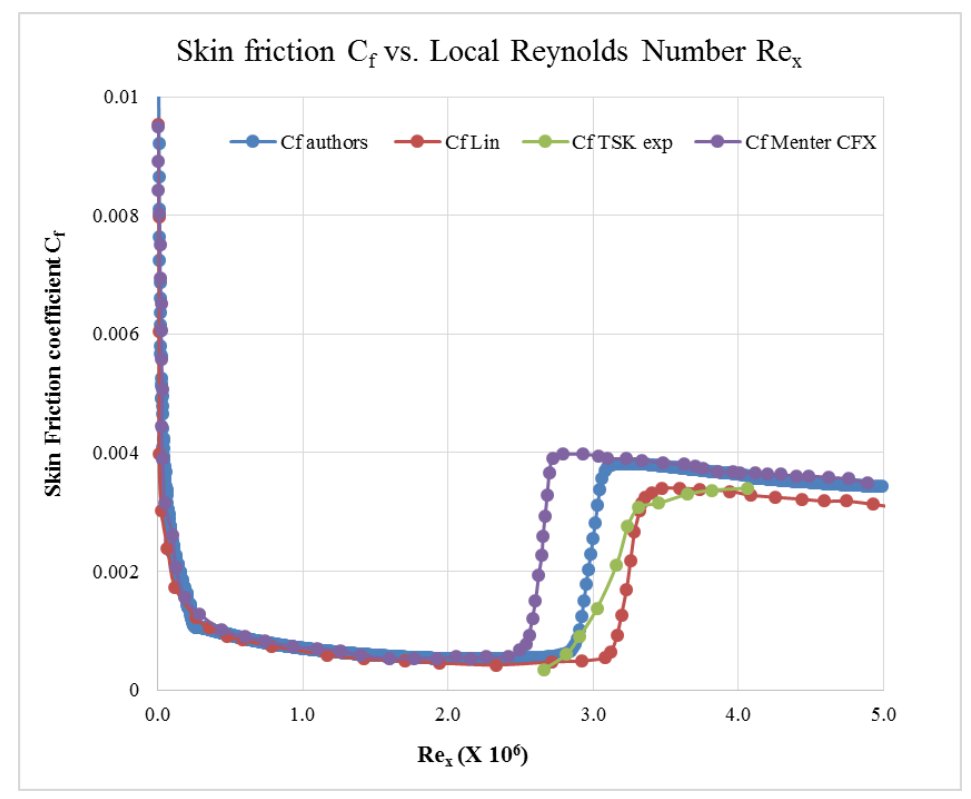

Figure 5 Benchmark graph of skin friction $C_{f}$ vs. local Reynolds number $\operatorname{Re}_{\mathbf{x}}$

\section{RESULTS}

The results in this section will be divided into two main subsections viz. the experimental results which show a comparison between three leading edges AR6, AR9 and AR12 and computational results which will be used to provide an insight into the flow structure around different AR leading edges. 


\subsection{Experimental Results}

Displacement thickness for the experimental case at AR12 and its comparison to the theoretically obtained Blasius displacement thickness is shown in Figure 6. The experimental displacement thickness closely matches the Blasius value. The displacement thickness at the end of the flat plate is roughly $1.49 \mathrm{~mm}$ for the experimentally obtained value while it is roughly $1.5 \mathrm{~mm}$ for the Blasius value. However, while there is a close correlation, transition onset cannot be ascertained. The shape factor $\mathrm{H}$ is used for ascertaining the transition onset in the boundary layer.

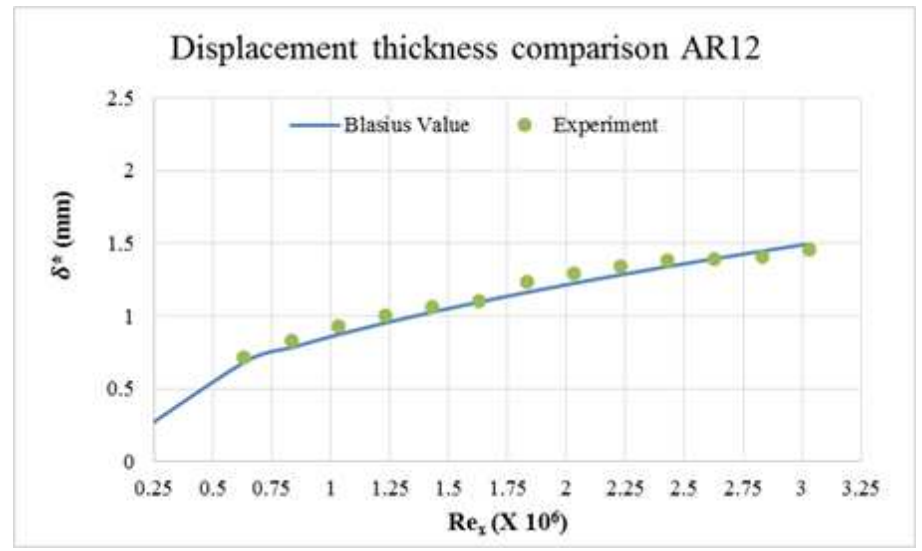

Figure 6 Displacement thicknesses comparison

The shape factor $\mathrm{H}$ tends to give an exact indicator of transition onset location and the length of the transition region. Theoretical calculations for the shape factor show that for transition onset in the boundary layer, the value for $H$ should be approximately 2.6. The point for the turbulent reattachment of the boundary layer is indicated by a shape factor value of $H=1.4$ (Apsley, 2009). As the shape factor reduces, the flow tends to indicate a highly turbulent flow structure. Castillo et al. (Castillo, et al., 2004) have defined the Intermittent Transitory Detachment position (ITD) at $H \sim 2.7$. The ITD position indicates that in the ITD the disturbances in the boundary layer start to amplify. The shape factors for AR6, 9 and 12 are shown in Figure 7 and are obtained from the experimental data. From (a), (b) and (c) in Figure 7, it can be seen that as expected in common sense transition onset is delayed as the AR is increased. The curve in (a) shows a rapid change in gradient at the transition onset point and the flow decays rapidly to turbulence. In the case of AR9 shown in (b), the change in the gradient is much lesser and the decay of the flow to turbulence in much more gradual indicating that the amplification of disturbances has reduced. For AR12 shown in (c), the length of the transition region is the largest indicating that the amplification of disturbances is the lowest and this can also be demonstrated by the turbulent spot propagation rates which are given in Table 1. 


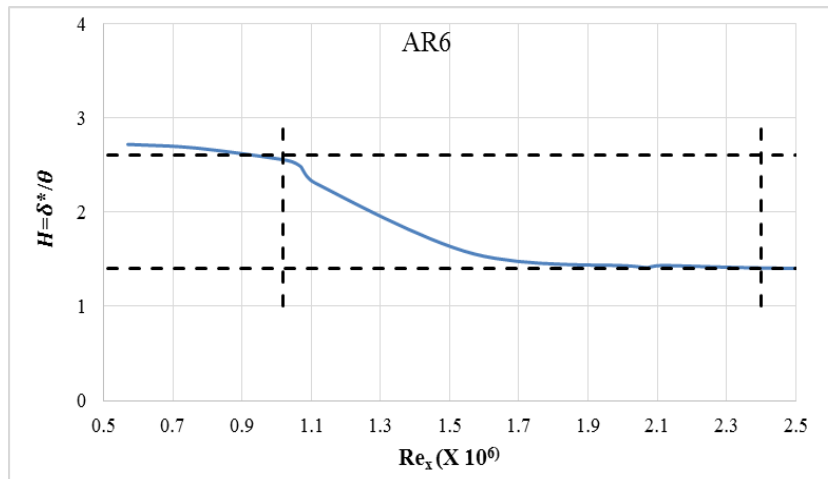

(a)

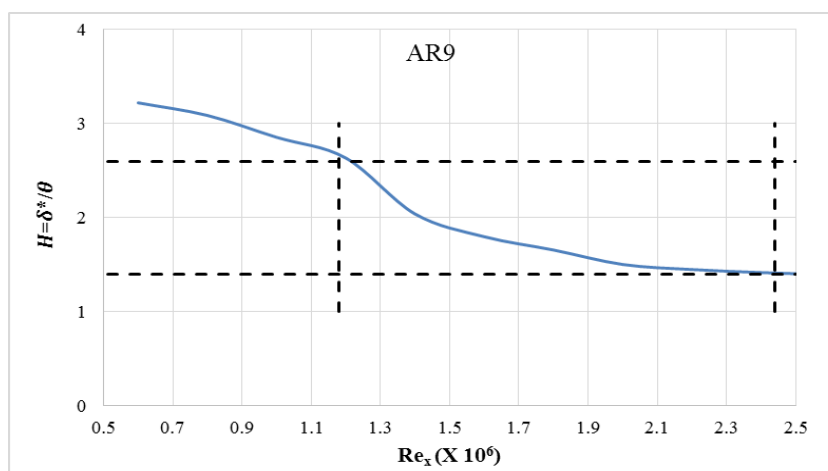

(b)

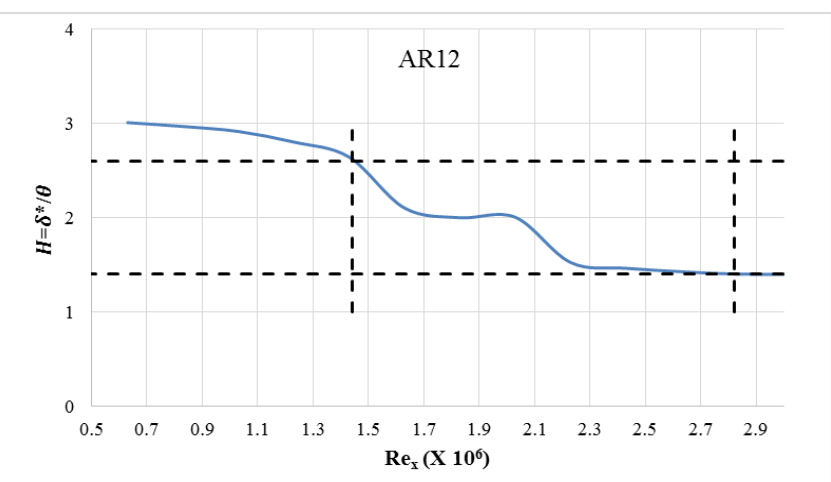

(c)

Figure 7 Shape factor $\mathrm{H}$ for different leading edges (Bhatia, et al., 2014)

Table 1 given below indicates the turbulent spot propagation rate for the experimental test cases. From the table, it can be seen that the turbulent spot propagation rate is clearly influenced by the change in leading edge geometry. At higher AR, the propagation rate drops sharply. The propagation rate has a direct impact on making the flow turbulent. Transition onset is delayed for AR12 as compared to other leading edges. In terms of experimental data, it can be observed that there is a significant difference between AR6 and AR12. The turbulent spot propagation rates indicate AR6 would have an extremely receptive boundary layer and amplification of disturbances takes place at a much faster rate. Figure $\mathbf{8}$ shows the percentage difference between the transition onset for AR6, 9 and 12. Transition onset for AR6 is $28 \%$ earlier than AR12 while it is $15 \%$ earlier for AR9. 
Table 1 Reynolds number and experimental spot propagation rates

\begin{tabular}{ccccc}
\hline $\mathbf{A R}$ & $\Delta_{\text {tr }} \mathbf{\%}$ & $\mathbf{R e}_{\text {tro }} \times \mathbf{1 0}^{\mathbf{6}}$ & $\mathbf{R e}_{\text {tre }} \times \mathbf{1 0}^{\mathbf{6}}$ & $\begin{array}{l}\widehat{\mathbf{n \sigma}} \\
\times \mathbf{1 0}^{-\mathbf{1 2}}\end{array}$ \\
\hline 12 & 0 & 1.42 & 2.82 & 1.3782 \\
9 & -15.49 & 1.20 & 2.42 & 1.7568 \\
6 & -28.16 & 1.02 & 2.40 & 2.5963 \\
\hline
\end{tabular}

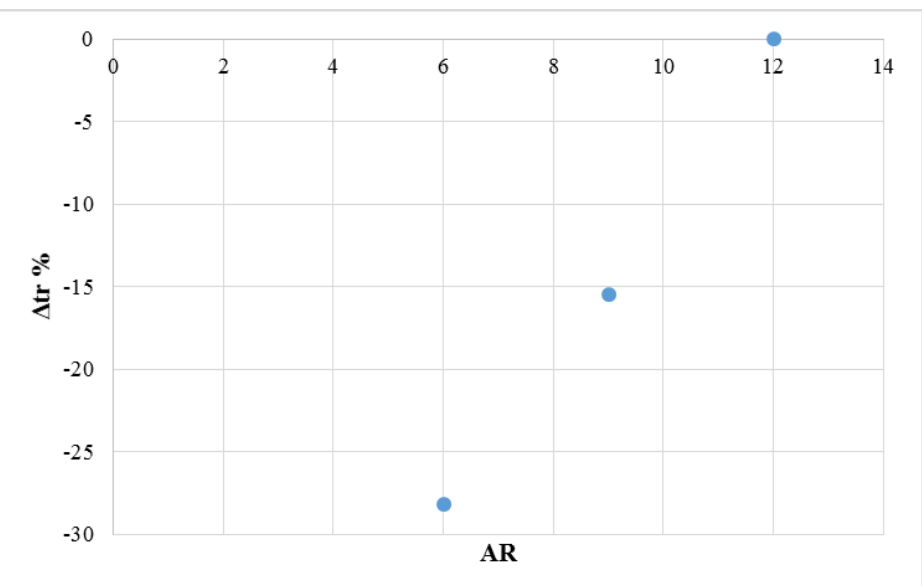

Figure 8 Experimental transition onset percentage difference between AR12, 9, 6

The main trend that emerges from the experimental results is that as the aspect ratio increases, the transition onset gets delayed. This corroborates with existing literature presented in Section 1.

\subsection{Computational Results}

The computational results presented in this subsection aims at highlighting the flow structure around different elliptical leading edges as well as highlight the performance differentials between higher AR leading edges that couldn't be tested experimentally. A plot of $C_{f}$ vs. the local Reynolds number $R e_{x}$, shown in Figure 9 , indicates that the transition onset follows a sequential pattern with the transition onset being delayed as the aspect ratio increases. An interesting trend to emerge from the plot is that there are three distinct groups of elliptical leading edges. The first group consists of the standalone AR4. The second group consists of AR6, AR9 and AR12 while the third group consists of the remaining two leading edges i.e. AR16 and AR20. The key outtake from this trend is that the performance deficit between AR6, 9 and 12 is not significant and there might be no justification in choosing one over the other.

However, there is a much larger gap between the transition onset Reynolds number of AR12 and AR16 while AR16 and AR20 have a very marginal performance deficit. Thus, there would be a strong case in choosing AR16 over AR12. However, the choice between AR16 and AR20 is a difficult one due to the relatively small performance differential. Also, it is to be noted that the transition onset Reynolds number for AR16 and AR20 are the closest to the transition onset Reynolds number for the ideal flat plate AR $\infty$. This indicates that AR16 and AR20 have superior aerodynamic performance as compared to the other elliptical leading edges. The difference between the transition onset location between $A R \infty$ and each individual leading edge as well as the spot propagation rates are given in Table 2 . 


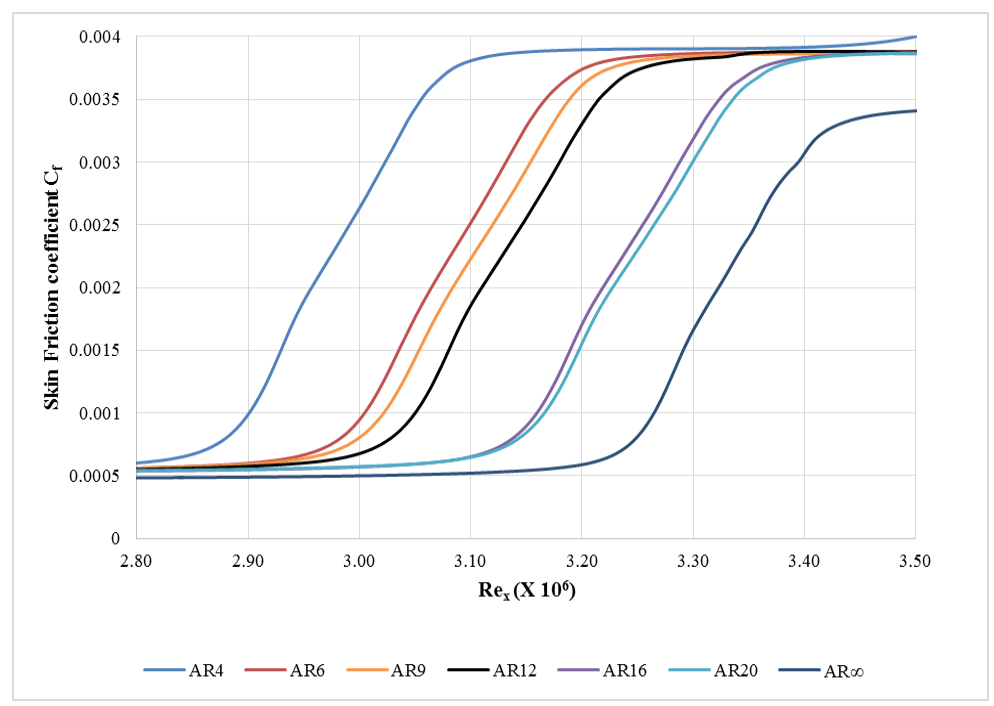

Figure $9 C_{f}$ values for $A R 4,6,9,12,16,20$ and $\infty$

Table 2 Transition onset location comparison for simulations

\begin{tabular}{lllll}
\hline $\begin{array}{l}\text { Aspect Ratio } \\
(\text { AR })\end{array}$ & $\Delta_{\text {tr }}(\%)$ & $\mathrm{Re}_{\text {tro }} \times \mathbf{1 0}^{\mathbf{6}}$ & $\mathrm{Re}_{\text {tre }} \times \mathbf{1 0}^{\mathbf{6}}$ & $\widehat{\boldsymbol{n}} \boldsymbol{\sigma} \times \mathbf{1 0}^{-\mathbf{1 1}}$ \\
\hline$\infty$ & 0 & 3.25 & 3.5 & 2.7767 \\
20 & -2.76 & 3.15 & 3.49 & 2.8004 \\
16 & -3.07 & 3.14 & 3.42 & 2.8025 \\
12 & -6.15 & 3.05 & 3.3 & 3.1644 \\
9 & -7.07 & 3.02 & 3.27 & 3.3309 \\
6 & -8.62 & 2.99 & 3.26 & 5.5502 \\
4 & -11.38 & 2.89 & 3.12 & 6.4029 \\
\hline
\end{tabular}

The turbulent spot propagation rates given in Table 2 further exemplify that AR20 demonstrates the lowest spot propagation rates of all elliptical leading edges. With all other parameters being constant in all test cases, it can be said that the spot propagation rate is inversely proportional to the aspect ratio of the leading edge. Higher AR leads to lower spot propagation rate and vice versa. Figure 10 shows the percentage difference between the transition onset for $A R \infty$ and other leading edges. It is assumed in this case that the transition onset $\%$ for $A R \infty$ is 0 and hence it is not reflected on the graph. A logarithmic equation has been used to give a rough estimate of the \% of early transition for different leading edges. This will enable researchers to choose an appropriate leading edge based on their requirement. The equation is as follows:

$$
\Delta t r \%=5.369 \ln (A R)-18.704
$$




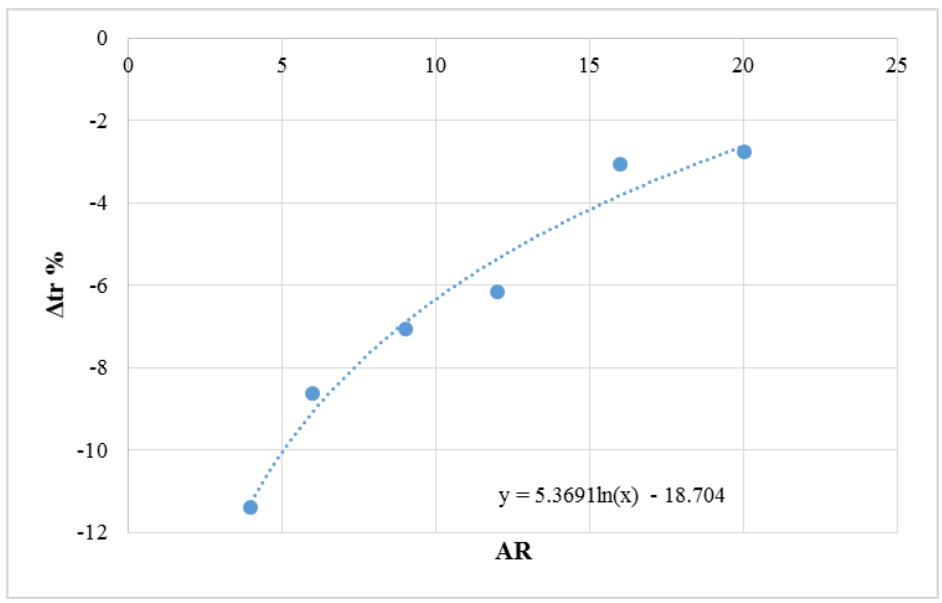

Figure 10 Transition onset percentage difference between $A R \infty$ and other leading edges

Thus, based on the accuracy requirement, researchers could choose from any of the three groups. High accuracy would require AR16 or AR20 while medium accuracy would tend to be a choice between AR6, 9 or 12. Low accuracy experiments/simulations could possibly use AR4. The trend for the transition onset location coincides with previously available experimental data i.e. AR20>AR16>AR12>AR9>AR6>AR4 (Bhatia, et al., 2014).

The results presented above, in absolute terms, give a good indicator of the impact of each leading edge. However, the results can be justified based on a flow structure analysis of each individual leading edge in isolation. In the present research case, all flow parameters are kept constant. The only variable in the entire simulation study is the curvature change of the elliptical leading edge. As each aspect ratio is characterised by a specific curvature, the curvature of each individual leading edge will change along the length of the leading edge and this will influence the flow structure along the leading edge. The presence of curvature discontinuities, as well as the nose of the leading edge, provides regions of high receptivity (Goldstein \& Hultgren, 1987). The other key region for receptivity is the leading edge/flat plate juncture (Hanson, et al., 2012). The pressure contours at the nose of the leading edge indicate that the region fore of the leading edge (the stagnation region) is greatly impacted by the presence of the leading edge as shown in Figure 11. However, the influence of the leading edge diminishes with an increase in the AR. The stagnation region is the largest for AR9 and the smallest for AR20. This can be further corroborated by evaluating the flow structure through the analysis of the pressure gradient $d P / d x$ for different leading edges shown in Figure 12. From Figure 12 (a), it can be observed that the adverse pressure increases with an increase in AR. However, it can also be observed that the pressure gradient tends to zero rapidly as the $A R$ increases. This is an indicator of the impact of the leading edge at the stagnation point. AR20 has the lowest peak favourable pressure gradient indicating the largest impact on the stagnation region albeit within a very small region. Conversely, AR9 has the largest favourable pressure gradient but with a larger stagnation region. AR $\infty$ being a plate with zero thickness and an infinite leading edge, the pressure gradient tends to remain constant with no stagnation point. Similarly, it can be observed in (b) that at the end of the leading edge, as the AR increases the adverse pressure gradient decreases. AR9 has the highest adverse pressure gradient while AR20 has the lowest adverse pressure gradient amongst all the elliptical leading edge. Higher adverse pressure gradient reduces the aerodynamic performance and this has a direct bearing on the transition onset location with all factors being constant. In the case of AR9, the pressure gradient changes from being the most favourable at the nose to being the most adverse leading edge/flat plate juncture. Thus, 
it can be assumed that as the flow progresses over the leading edge, it is greatly impacted by changes in the geometry. Quite conversely, in the case of AR20, the flow progresses from having the most adverse pressure gradient to the most favourable pressure gradient at the end of the leading edge once again highlighting the impact of geometric changes on the flow structure. The geometric changes in all the leading edges appear in the form of changes in the curvature.
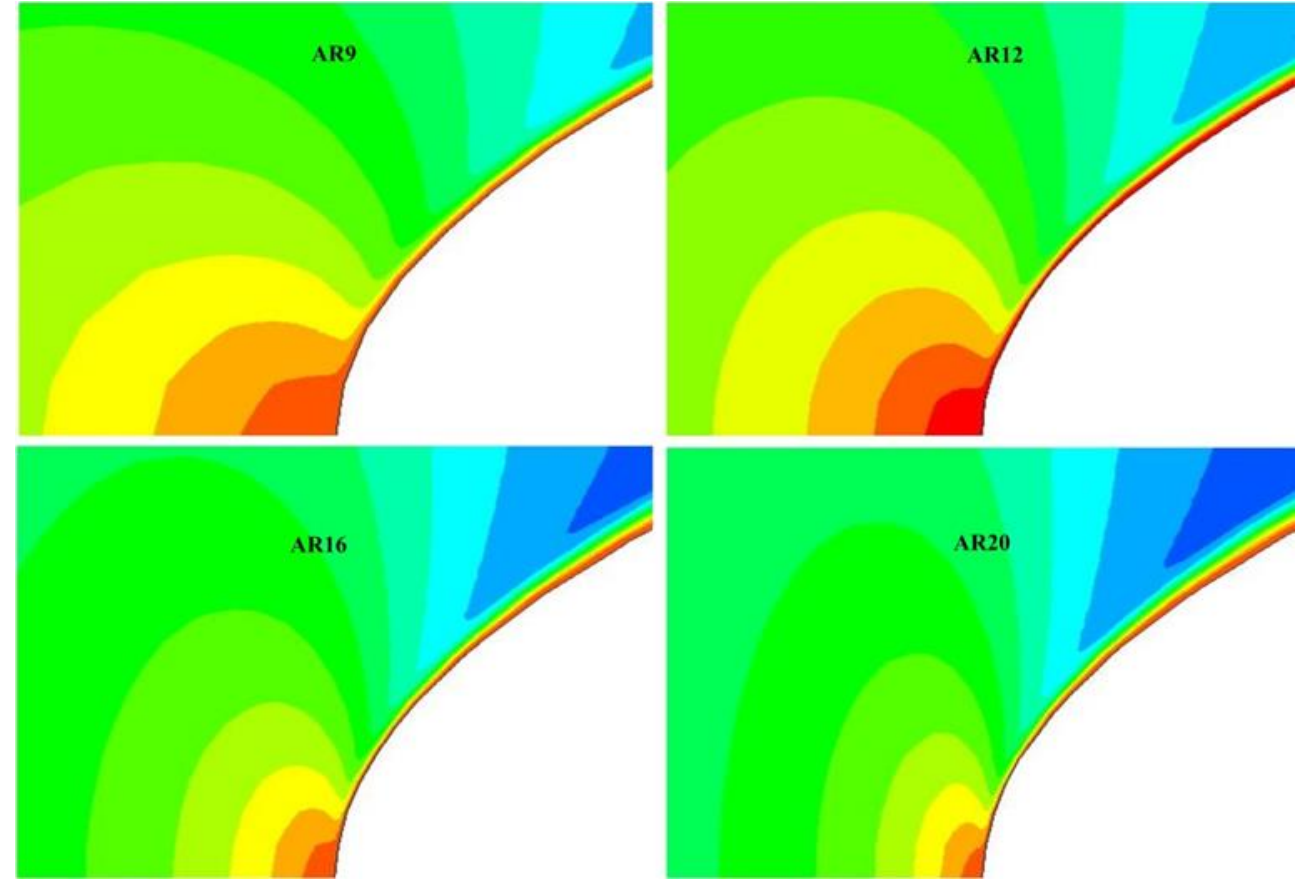

Figure 11 Pressure contours at the stagnation region for different leading edges

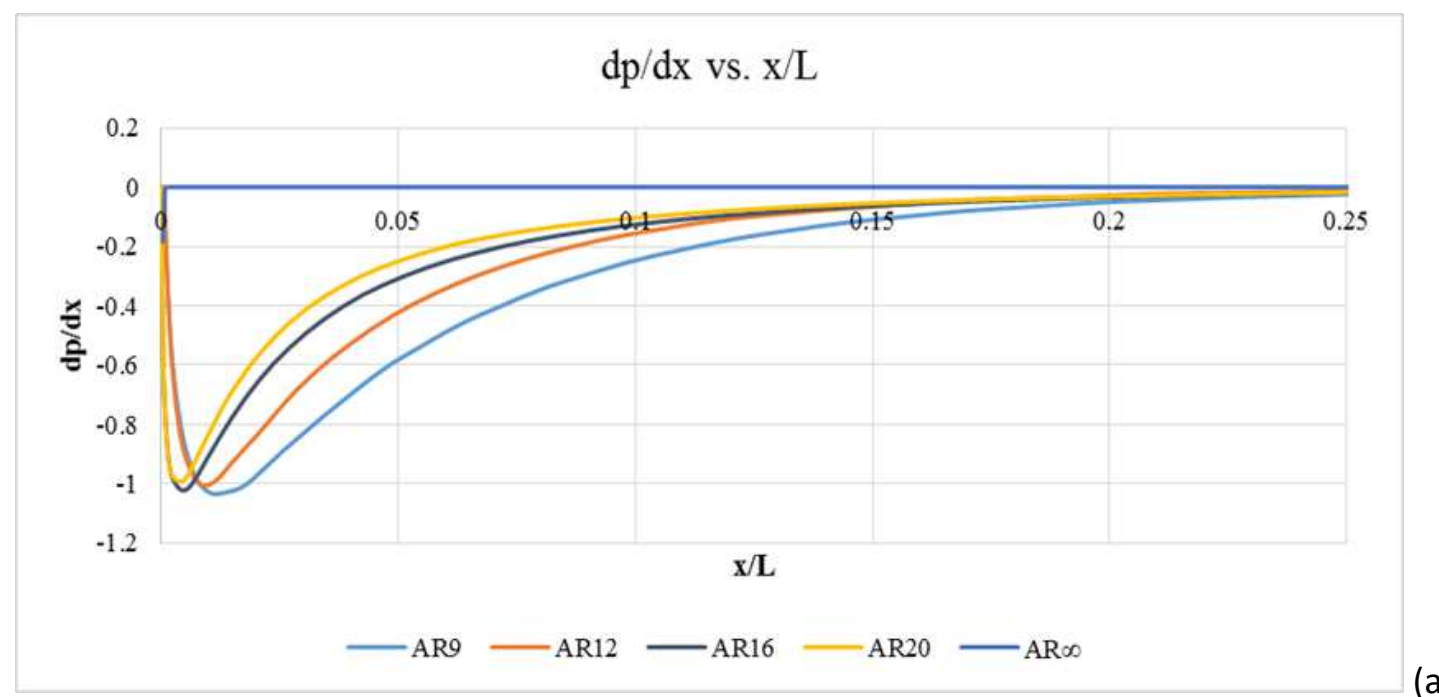

(a) 


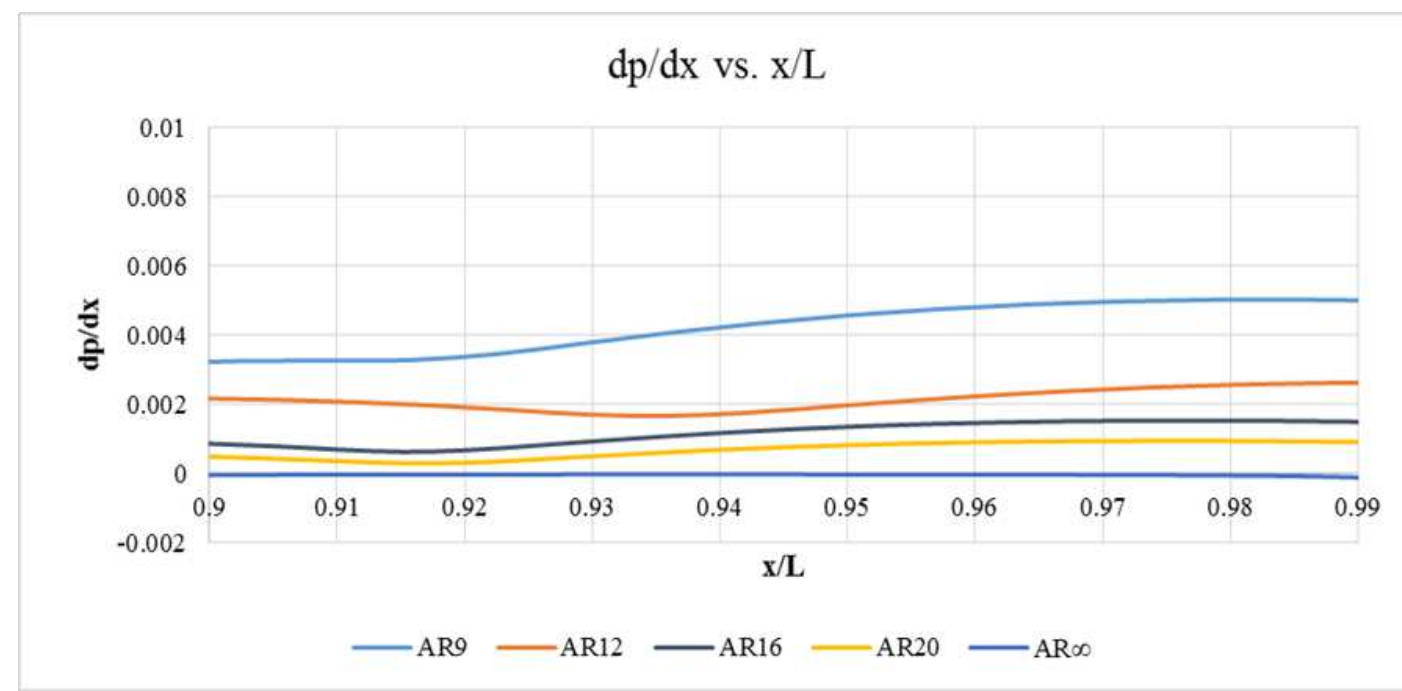

(b)

Figure 12 Pressure gradient for different leading edges

To fully understand the impact of changes in curvature, initially, the curvature of each individual leading edge is plotted along the length of the leading edge. Figure 13 displays the curvature ' $\mathrm{k}$ ' along the length of the leading edge. From the figure, it is evident that that for each individual leading edge, the maximum curvature is at the start of the leading edges as shown in (a). Amongst all the leading edges, AR20 has the highest curvature at the start i.e. the nose of the leading edge. The curvature at the start decreases with a decrease in AR. Thus, the detrimental effect on the flow structure at the nose due to an increase in AR can be explained with the higher curvature values. At $x / L=0.05$., AR 9 has the largest curvature. The curvature decreases with an increase in AR. By $x / L=0.5$, AR 16 and AR20 show a very gradual curvature change and tend to indicate curvature values closer to the ideal leading edge with $A R \infty$ as shown in (b). By the end of the leading edge i.e. at $x / L=1$, $A R 20$ has a curvature value which is closest to $A R \infty$.

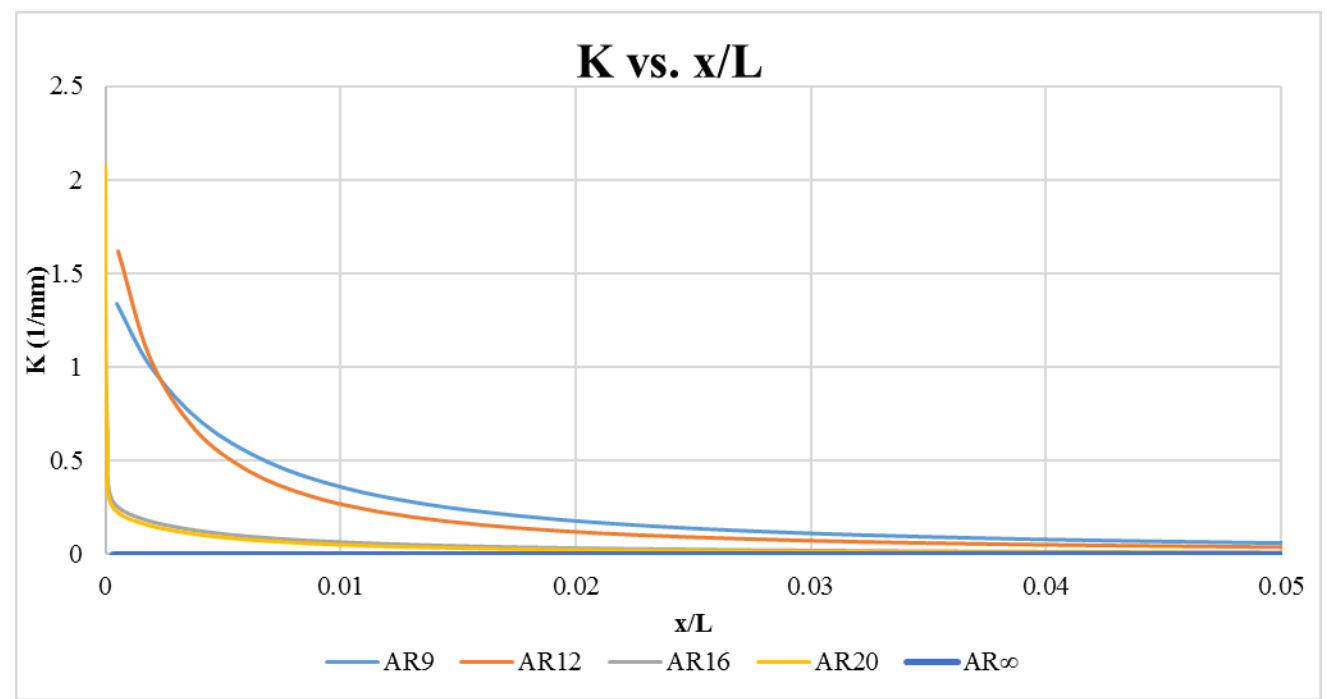

(a) 


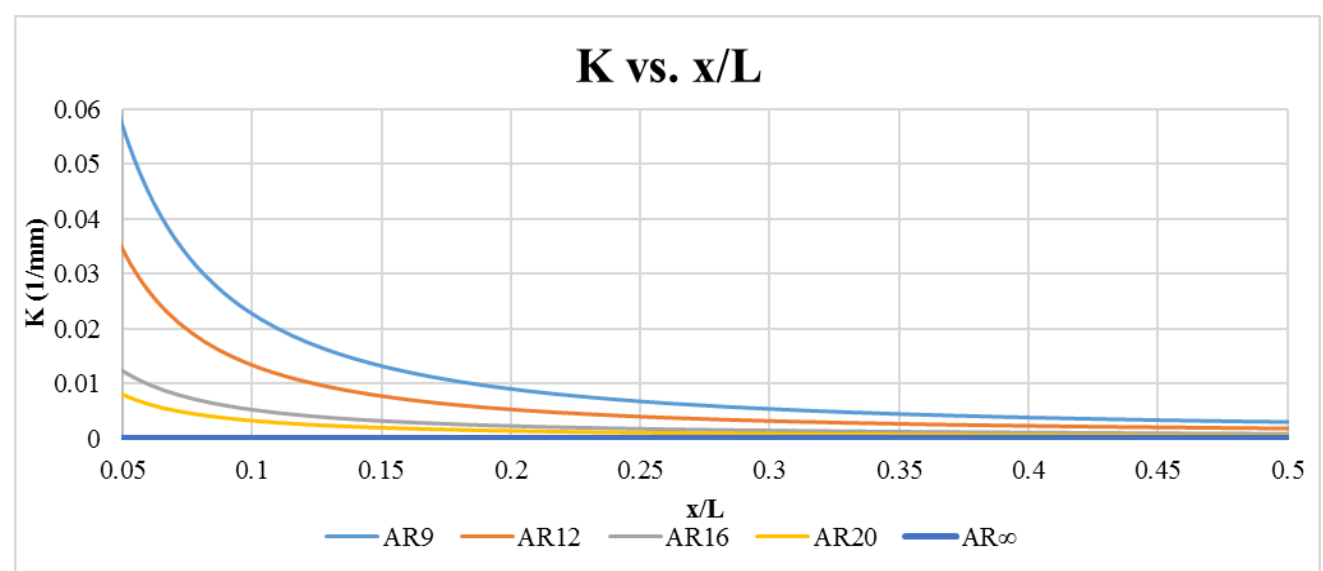

(b)

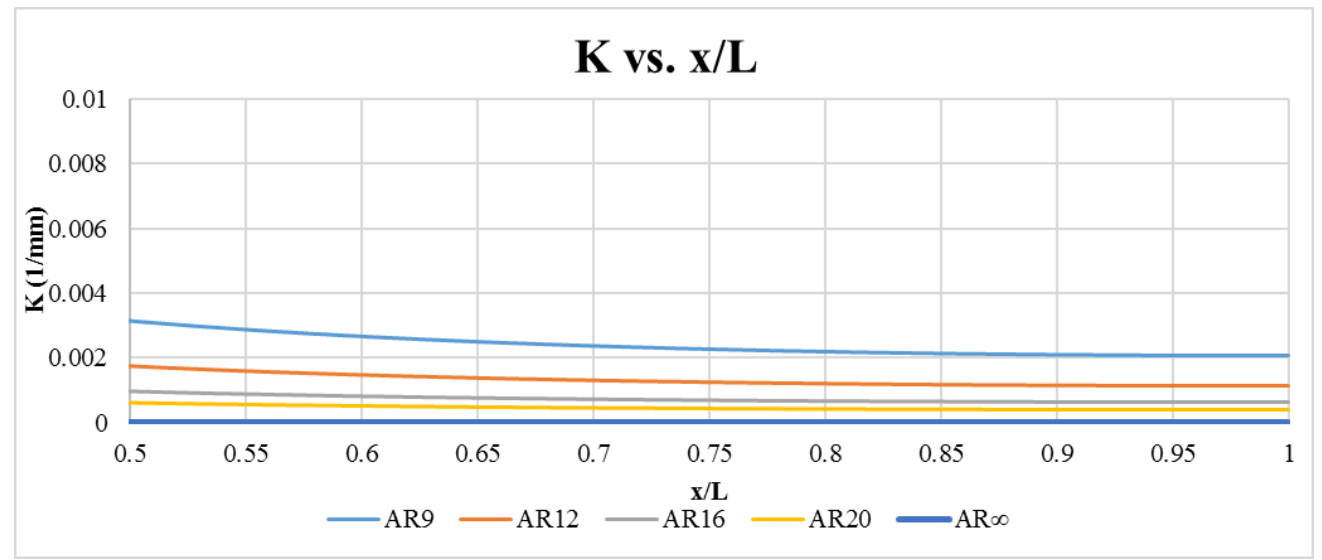

(c)

Figure 13 Curvature along the length of leading edge for different AR

The change in curvature $d k$ will further highlight the differences between different aspect ratio leading edges and is shown in Figure 14. The curvature change is plotted along the length of the curve $d s$ which is calculated using the following formula:

$d s=\sqrt{(d x)^{2}+(d y)^{2}}$

From Figure 14, it can be observed that at the start of the leading edge, the curvature change is more drastic for the higher AR leading edge such as AR16 and AR20. AR 9 and AR12 show a more gradual change in the curvature along the length of the leading edge. As the $A R$ increases, the rate of change of curvature reduces along the length of the leading edge. This indicates that, as the flow progresses over the leading edge, the influence of curvature changes diminish with an increase in AR. These curvature changes are high receptivity areas for disturbances to enter the boundary layer. Thus, the impact of a higher AR leading edge is to reduce the receptivity of the boundary layer to disturbances owing to a reduction in curvature discontinuities. 


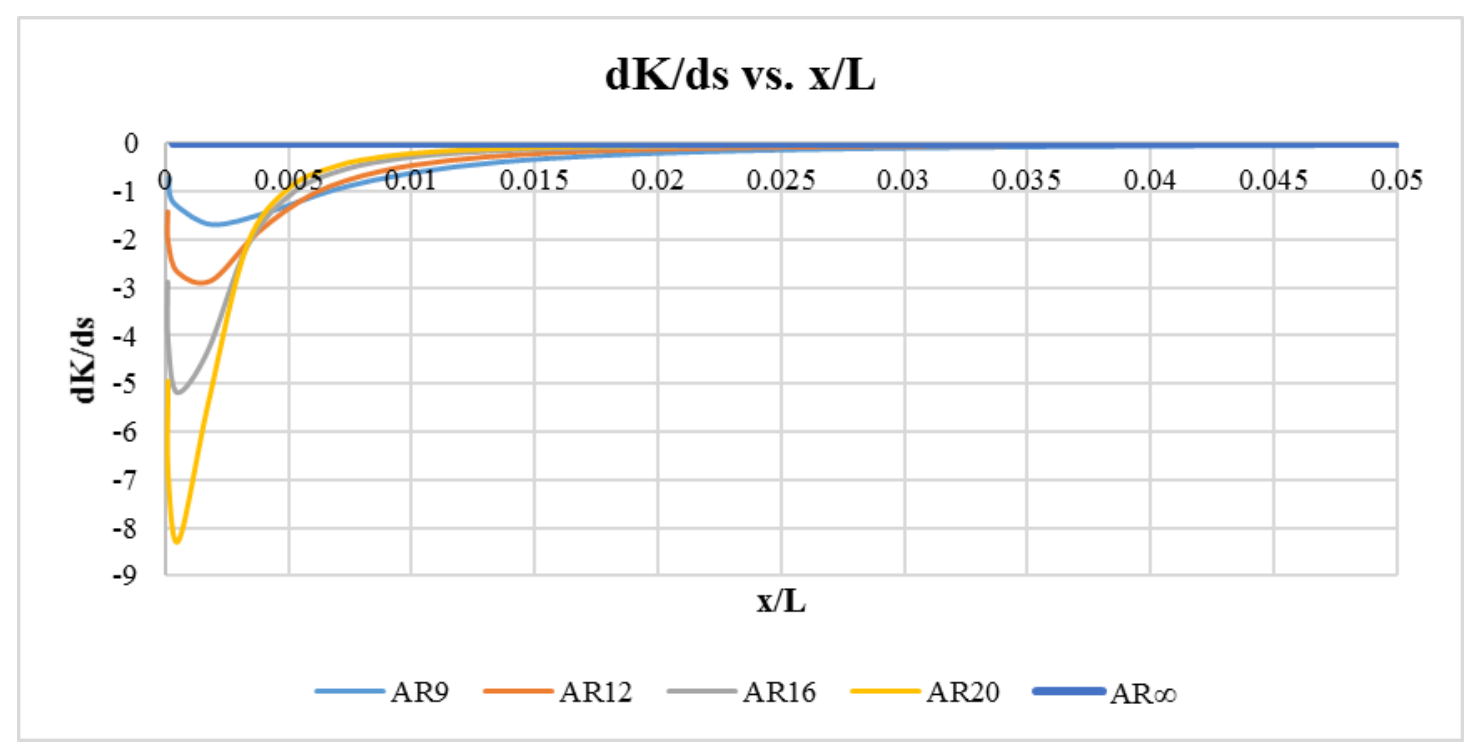

Figure 14 Curvature change along the length of leading edge for different AR

To further analyse the impact of the leading edge on the receptivity of the boundary layer, a plot of $C p^{*}$ vs. $x / L$ is shown in Figure 15. $C p^{*}$ represents the ratio of the $C p$ of each individual leading edge to that of $A R \infty$.

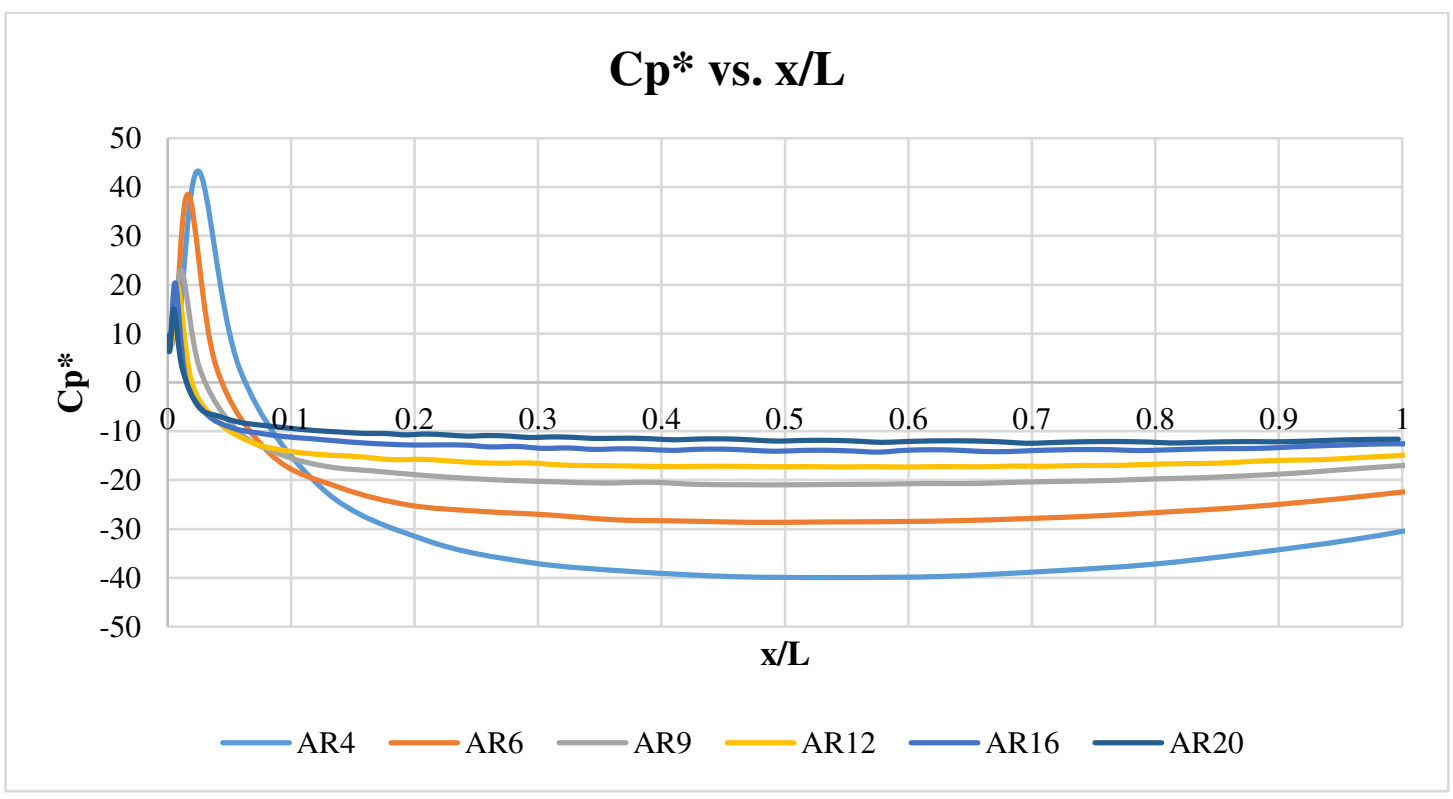

Figure $15 \mathrm{Cp} *$ vs. $\mathrm{x} / \mathrm{L}$ for different leading edges

The variable $C p^{*}$ can be used to perform a comparative analysis between the relative performances of each individual leading edge. From Figure 15, it can be observed that, as compared to the ideal leading edge, AR4 has a very high peak $C p^{*}$ value. This value reduces with an increase in the AR. From the data for AR20, it can be observed that the impact of AR20 is the least and has a relatively low $C p^{*}$ value indicating that its performance is closer to that of $A R \infty$. Also, the data indicates that the peak value for AR20 is obtained very close to the start of the leading edge whereas the peak values for other leading edges are obtained sequentially later along the leading edge as the AR decreases. This could possibly indicate that the impact of the curvature extends for a larger area on the leading edge which could have an impact the receptivity of the flow. It can also be observed that as the AR increases, $C p^{*}$ tends to indicate a constant ratio along the length of the leading edge particularly in the case of the 
AR16 and AR20. This indicates that the influence of the curvature diminishes and its impact of the flow stagnates beyond a certain point on the leading edge.

\subsection{The case of the wedge vs. the ellipse}

The preceding sections discuss the performance of different elliptical leading edges. One of the key observation from the result is that the aerodynamic performance of the leading edge increases with an increase in AR. However, in terms of practical experimental scenarios, higher AR leading edges such as AR16 and AR20 might be difficult to manufacture. To justify the use of an elliptical leading edge, it is essential to compare it with the simplest leading edge geometry i.e. the wedge. A wedge having the same length as AR20 is used and the performance of the wedge is compared against AR20 and AR $\infty$. A case has been presented in this section to compare a wedge having the same dimensions as that of an AR20 elliptical leading edge. In the case of the wedge, the two areas of discontinuities that would introduce disturbances in flow would be at the nose and at the leading edge-flat plate juncture. The leading edge-flat plate juncture could possibly have the highest impact owing to a sharp edge at the leading edge flat plate juncture.

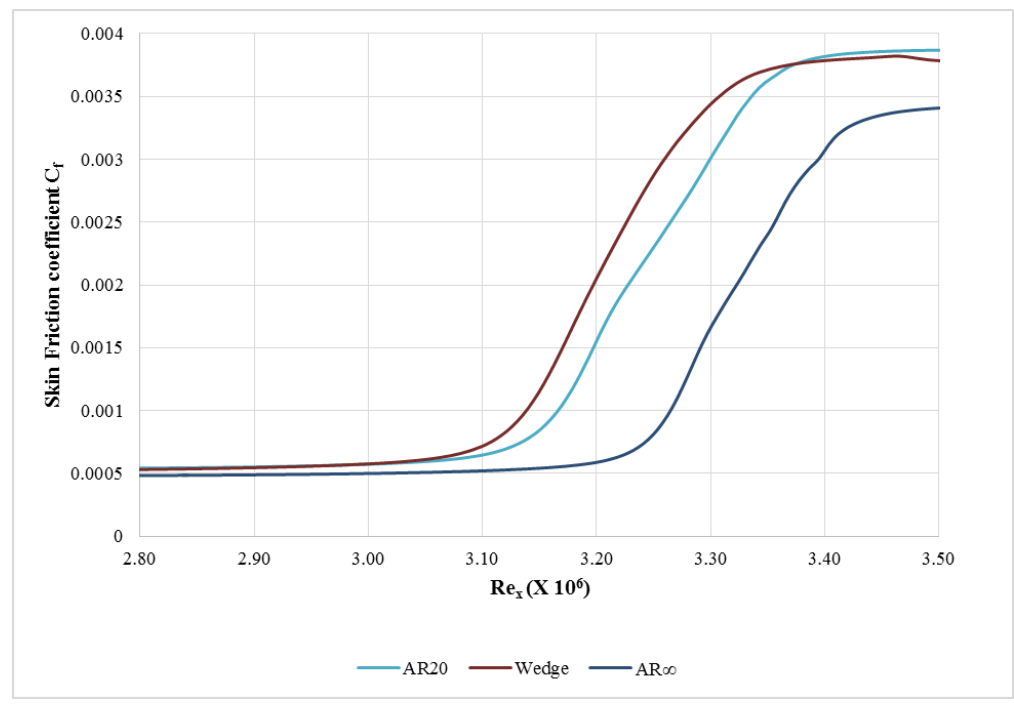

Figure 16 Skin friction coefficient for Wedge against AR20 and AR $\infty$

Table 3 Transition onset comparison between Wedge, AR20, and AR $\infty$

\begin{tabular}{ccccc}
\hline $\begin{array}{l}\text { Aspect Ratio } \\
\text { (AR) }\end{array}$ & $\boldsymbol{\Delta}_{\text {tr }}(\boldsymbol{\%})$ & $\mathbf{R e}_{\text {tro }} \times \mathbf{1 0}^{\mathbf{6}}$ & $\mathbf{R e}_{\text {tre }} \times \mathbf{1 0}^{\mathbf{6}}$ & $\widehat{\boldsymbol{n}} \boldsymbol{\sigma} \times \mathbf{1 0}^{\mathbf{- 1 1}}$ \\
\hline$\infty$ & 0 & 3.25 & 3.5 & 2.7767 \\
20 & -2.76 & 3.15 & 3.49 & 2.8004 \\
16 & -3.07 & 3.14 & 3.42 & 2.8025 \\
Wedge & -4.92 & 3.09 & 3.48 & 3.0688 \\
12 & -6.15 & 3.05 & 3.3 & 3.1644 \\
9 & -7.07 & 3.02 & 3.27 & 3.3309 \\
6 & -8.62 & 2.99 & 3.26 & 5.5502 \\
4 & -11.38 & 2.89 & 3.12 & 6.4029 \\
\hline
\end{tabular}

Table 3 provides enough evidence as to the merits of having an elliptical leading edge. The pressure gradient along the length of the leading edge shows that while, the wedge has a very small stagnation region the favourable pressure gradient for the wedge reduces very rapidly and tends to zero after the stagnation region as shown in Figure 17. The impact of the elliptical leading edge is to maintain the favourable pressure gradient for longer. 


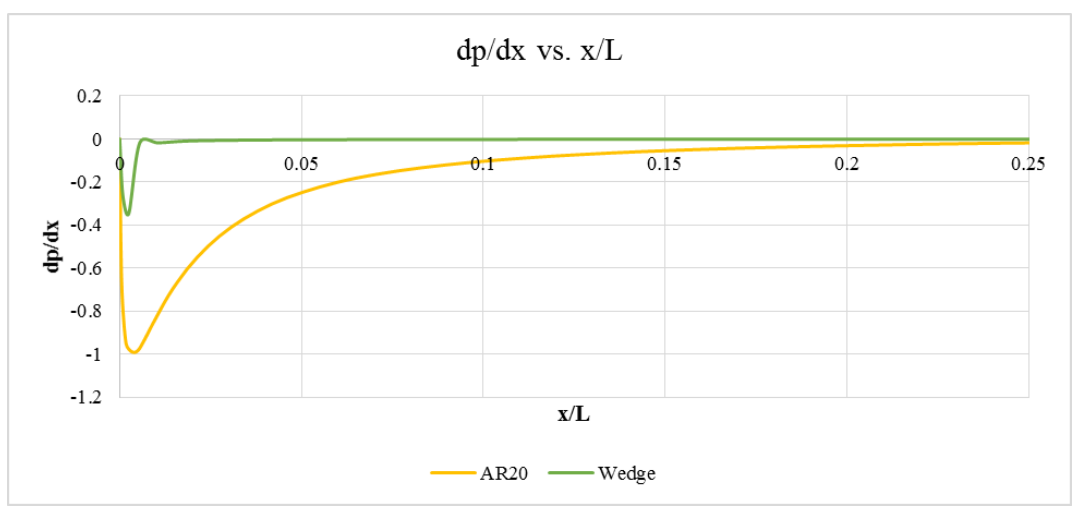

Figure 17 Pressure gradient for Wedge and AR20

A plot of the $C p^{*}$ vs. $x / L$ for the wedge along with its comparison to different elliptical leading edges is shown in Figure 18. An analysis of the plot indicates that at the start of the leading edge, the wedge has the smallest peak $C p^{*}$ value indicating that its impact on the flow at the start is closer to $A R \infty$ as compared to the elliptical leading edges. For a majority of the length of the leading edge, the wedge maintains a competitive advantage over the elliptical leading edges. However, by $x / L=0.85, C p^{*}$ for the wedge is comparable to AR20 and AR16. By $x / L=1$, the wedge shows $C p^{*}$ values that are comparable to AR6. The trend for the wedge indicates that while, for a majority of the length of the leading edge, it tends to hold a performance advantage over other leading edges, by the end of the plate its advantage diminishes rapidly and the elliptical leading edges tend to perform better. The advantage that the wedge has over the other AR leading edges over a majority of the leading edge ultimately enables it to have a performance that is better than AR12 but worse than AR16.

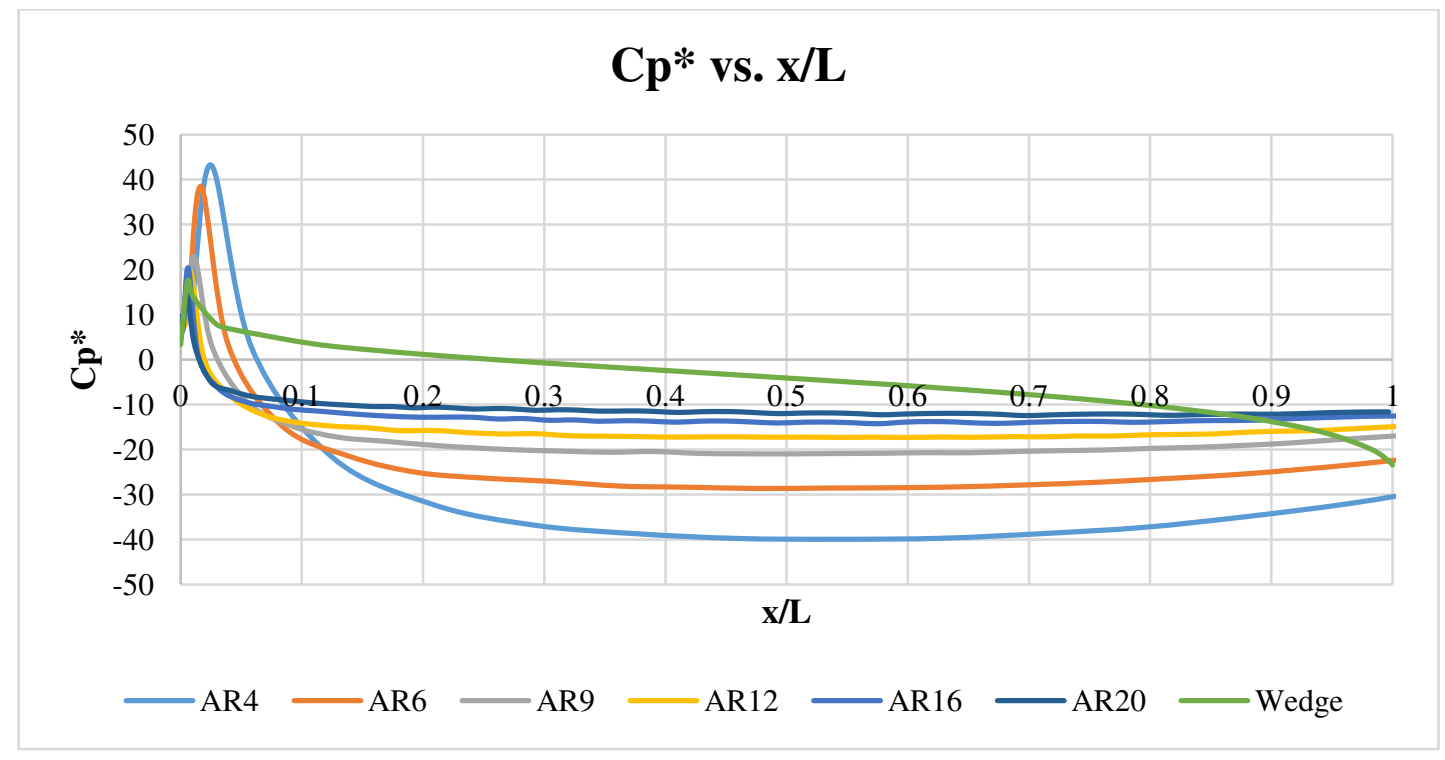

Figure 18 Comparison of $\mathrm{Cp}^{*}$ vs. $\mathrm{x} / \mathrm{L}$ for the wedge against different elliptical leading edges

\section{DISCUSSIONS}

The results presented in Section 3 demonstrate that as the aspect ratio increases transition is delayed. While all other parameters are constant, the impact of the leading edge can be narrowed down to its curvature. The changes in the curvature along the leading edge plays a significant impact on the transition onset point. The presence of an adverse pressure gradient, 
as well as the turbulent spot propagation rates, are a consequence of changes in curvature. From the $\Delta$ tr values given in Table 1, it is evident that for experimental tests, AR12 is the most popular choice amongst all the leading edges tested. AR9 has an early transition onset by $15 \%$ and AR6 has an early transition of $28 \%$. From the standpoint of accuracy, it would inadvisable to recommend AR6 and below for any experimental investigations barring test work required to do some sample data collection. AR9 could still be used for experiments that do not require a high level of accuracy in transition onset. AR12 would be the ideal leading edge for experimental tests and is the authors are of the opinion that it is the optimum choice in the current test scenario. However, at the same time, the ability to easily machine the lower AR leading edges tends to provide the flexibility required by researchers to conduct experiments to achieve approximate transition onset results. Further experimental tests would be required to study the feasibility of higher AR such as AR14, 16 and 20 owing to the difficulties in manufacturing them.

At the same time, simulations show that AR16 and AR20 have better performance as compared to AR9 and AR12. The difference between AR16 and AR20 is a mere $0.3 \%$ while the difference between AR9 and AR20 is roughly $4 \%$ as shown in Table 2 . When compared to the ideal case scenario of $A R \infty, A R 12$ has an earlier transition onset by roughly $6.2 \%$. In the case of AR16 and AR20, this is roughly 3\%. The impact of AR20 is that it has the highest adverse pressure gradient at the stagnation region, but its effect is confined to a very small region.

The rate of curvature change $d K / d s$ and the pressure gradient $d P / d x$ are interlinked and it can be observed that, in the case of AR20, the curvature is rapid in the beginning after which it stabilises. It is well known, that the receptivity of the boundary increases with an increase in curvature discontinuities. In the case of AR20, the sharp change in curvature is confined to only to the start of the leading edge after which the rate of curvature change is gradual. Conversely, in the case of AR9, the curvature change at the start of the leading edge is more gradual and the rate of curvature change doesn't approach a constant value until very late on the leading edge thereby having a larger influence on the flow structure. This tends to have a knock on effect on the transition onset location. The logarithmic function given in Figure 10 can be used to predict the transition offset between $A R \infty$ and a particular leading edge. This would give prospective researchers a choice of an appropriate AR leading edge based on their research objectives. Researchers requiring a high degree of accuracy can choose a higher AR leading edge such as AR16 while experimental work having a higher tolerance could possibly use AR9 and AR12 as the difference to the ideal case scenario is varies in the rage of 7-15\%.

The wedge discussed in Section 3.3 also demonstrates that its performance is better than AR9 and AR12 but worse than AR16 and AR20. This could potentially give a choice between choosing an elliptical leading edge or a wedge, based on the application. The wedge has an advantage owing to its simplistic geometric configuration which allows for easy manufacture and maintenance. Further study is needed to find the optimum leading edge for all test cases. A potentially optimal solution would involve a wedge-ellipse combination as the wedge has a small stagnation region and the elliptical leading edge has better fluid flow properties at the leading edge-flat plate juncture.

\section{CONCLUSION}

The effects of different leading edges on boundary layers subjected to a transitional regime at low free stream turbulence have been studied comprehensively and documented. The impact of changes in the aspect ratio of the elliptical leading edge is quite significant. The presence of a higher aspect ratio tends to delay transition onset. In terms of transition onset 
location, it can be concluded that AR20>AR16>AR12>AR9>AR6>AR4. However, at the start of the leading edge, the lower $A R$ tend to have a larger stagnation region albeit with a lower impact on the fluid. As the flow develops over the leading edge, an increase in AR leads to better aerodynamic performance. From the present set of results, it can be concluded that while AR20 is the best elliptical leading edge, AR16 would be the most optimum choice as the difference in performance is negligible as compared to AR20 but better than AR12. AR16 would be easy to machine as well. However, the presence of a wedge-shaped leading presents a strong case as an alternative to an elliptical leading edge. The authors recommend the use of wedge-shaped leading edge instead of lower AR leading edges in most experimental test scenarios. The choice of the leading edge is a trade-off between machinability and accuracy.

\section{DECLARATIONS}

Availability of data and materials

The data and materials for this manuscript have been embedded in the form of spreadsheets within the manuscript itself. Datasets can be provided where necessary.

\section{Competing interests}

Not Applicable

\section{Funding}

The authors wish to thank the Ningbo Science \& Technology Bureau for their funding under the Ningbo Natural Science Programme Project having project code: 2019A610116. The authors would also like to thank the University of Nottingham Ningbo China for their funding under the Faculty of Science and Engineering's New Researcher Grant (NRG).

\section{Authors' contributions}

Dr Dinesh Bhatia performed the Experimental Wind Tunnel Tests and the CFD simulations for the project. Dr Guangjun Yang provided expertise for the Wind Tunnel Tests. Dr Guangning Li provided expertise for the CFD simulations. Prof Jian Wang provided his expertise of Aerodynamics and steered the research in the appropriate direction.

\section{Acknowledgements}

We thank Northwestern Polytechnical University, Xi'an, China for allowing us the unrestricted use of the Low Turbulence Wind Tunnel in their premises. We also thank the University of Nottingham Ningbo China and Kingston University London for providing facilities and encouragement to perform the CFD simulations. 


\section{REFERENCES}

Apsley, D. D., 2009. [Online]

Available at:

http://personalpages.manchester.ac.uk/staff/david.d.apsley/lectures/turbbl/integral.pdf

Bhatia, D. D., 2016. Effects of surface features and vibration of flat plate on boundary layer transition, London: Kingston University.

Bhatia, D. D., Yang, G., Sun, J. \& Wang, J., 2014. Effect of Leading Edge Geometry on Boundary Layer Transition-An Experimental Approach. Applied Mechanics and Materials, Volume 590, pp. 53-57.

Castillo, L., Wang, X. \& George, W. K., 2004. Separation Criterion for Turbulent Boundary Layers Via Similarity Analysis. Journal of Fluid Engineering, Volume 126, pp. 297-303.

Dantec Dynamics, 2013. Dantec Dynamics News. [Online]

Available at: http://www.dantecdynamics.com/docs/news-and-

events/PI 313 MiniCTA MiniCTA package and MultiChannel CTA versions.pdf

[Accessed 2701 2014].

Goldstein, M. E. \& Hultgren, L. S., 1987. A note on the generation of Tollmien-Schlichting waves by sudden surface-curvature change. J Fluid Mech, Volume 181, pp. 519-525.

Görtler, H., 1941. Über eine dreidimensionale Instabilität laminarer Grenzschichten an konkaven, Göttingen: Wänden Gesellschaft der Wissenschaften zu Göttingen, Nachrichten.

Hanson, R. E., Buckley, H. P. \& Lavoie, P., 2012. Aerodynamic optimization of the flat-plate leading edge for experimental studies of laminar and transitional boundary layers. Exp Fluids, Volume 53, pp. 863-871.

Kendall, J., 1991. Studies on laminar boundary-layer receptivity to freestream turbulence near a leading edge. ASME, pp. 23-30.

Klingmann, B., Boiko, A. W. K., Kozlov, V. \& Alfreddson, P., 1993. Experiments on the stability of Tollmien-Schlichting waves. Eur. J. Mech. /B Fluids, Volume 12, pp. 493-514.

Langtry, R. et al., 2004. A Correlation-Based Transition Model Using Local Variables-Part II: Test Cases and Industrial Applications. J. Turbomach, 128(3), pp. 423-434.

Lin, Y., Raghunathan, S., Raghunathan, B. \& Mcllwain, S., 2012. Prediction of boundary layer transition on a flat plate subject to surface waviness. Journal of Aerospace Engineering, 226(1), pp. 42-54.

Malan, P., Suluksna, K. \& Juntasaro, E., 2009. Calibrating the Y-ReO Transition Model for Commercial CFD. s.l., s.n.

Mayle, R., 1991. Laminar-Turbulent Transition in Gas Turbine Engines. Journal of Turbomachinery, 133(4), pp. 509-537.

Menter, F., Langtry, R. \& Völker, S., 2006. Transition modelling for general purpose CFD codes. Flow, Turbulence and Combustion, Volume 77, pp. 277-303.

Nagarajan, S., Lele, S. \& Ferzigeer, J., 2007. Leading-edge effects in bypass transition. J.Fluid.Mech, Volume 572, pp. 471-504. 
Saric, W., White, E. \& Reed, H., 1999. Boundary-Layer Receptivity to freestream disturbances and its role in Transition (Invited). Norfolk, VA, s.n., pp. 1-18.

Schraub, F. \& Kline, S., 1965. Rept. MD-12, s.I.: s.n.

Schubauer, G. \& Klebanoff, P., 1955. Contribution on the mechanics of boundary layer transition, s.I.: NACA.

Schultz, M. P. \& Volino, R. J., 2003. Effects of Concave Curvature on Boundary Layer Transition under High Freestream Turbulence Conditions. ASME, January, Volume 125, pp. 18-27.

Weisstein, E. W., 2015. Curvature. [Online]

Available at: http://mathworld.wolfram.com/Curvature.html

[Accessed 0809 2015].

Weisstein, E. W., 2015. Ellipse. [Online]

Available at: http://mathworld.wolfram.com/Ellipse.html

[Accessed 0809 2015].

Westin, K. et al., 1994. Experiments in a boundary layer subjected to free stream turbulence. Part 1. Boundary layer structure and receptivity. J.Fluid Mech, Volume 281, pp. 193-218.

Zaki, T. \& Durbin, P., 2006. Continuous mode transition and the effects of pressure gradient. J.Fluid Mech, Volume 563, pp. 357-388. 
Figures

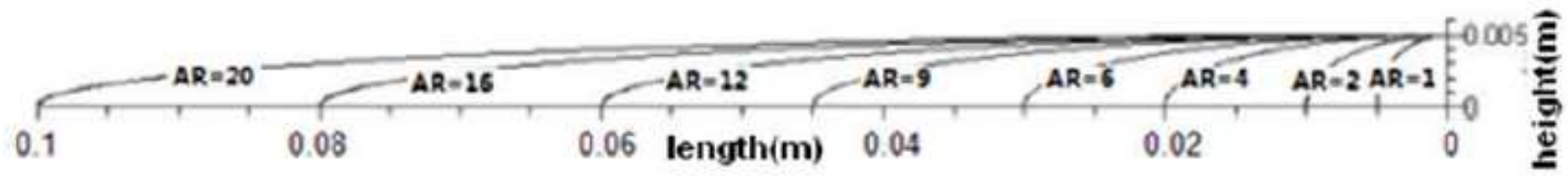

Figure 1

Individual profiles for different AR leading edges

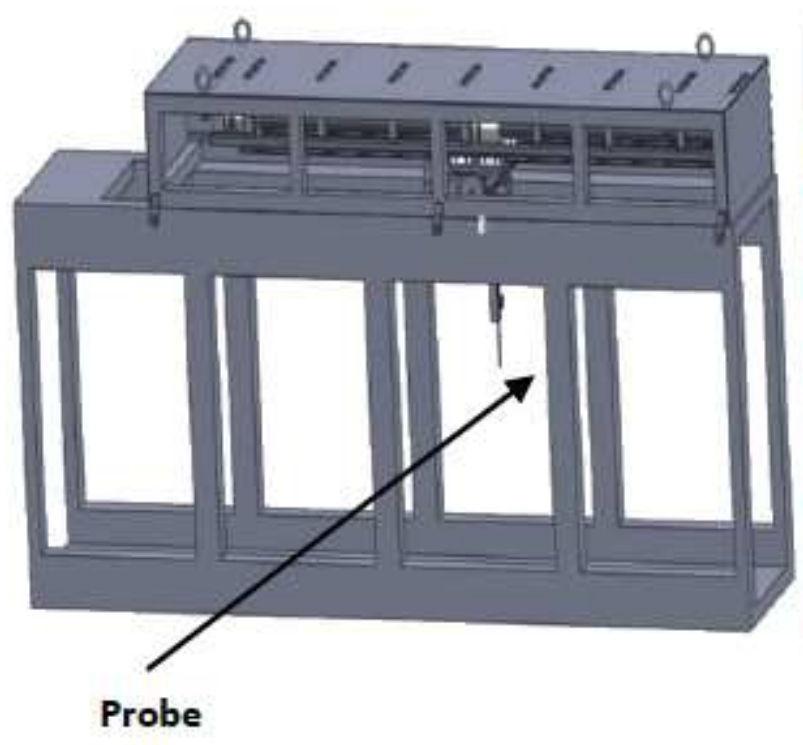

Figure $\mathbf{2}$ Schematics of the test assembly

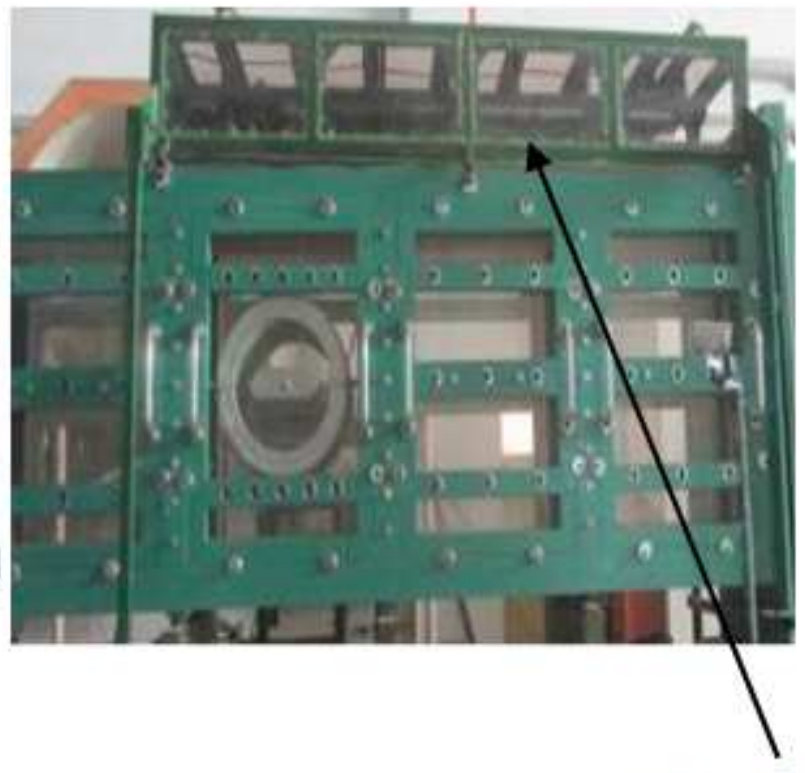

Linear Traverse

Figure 2

Schematics of the test assembly

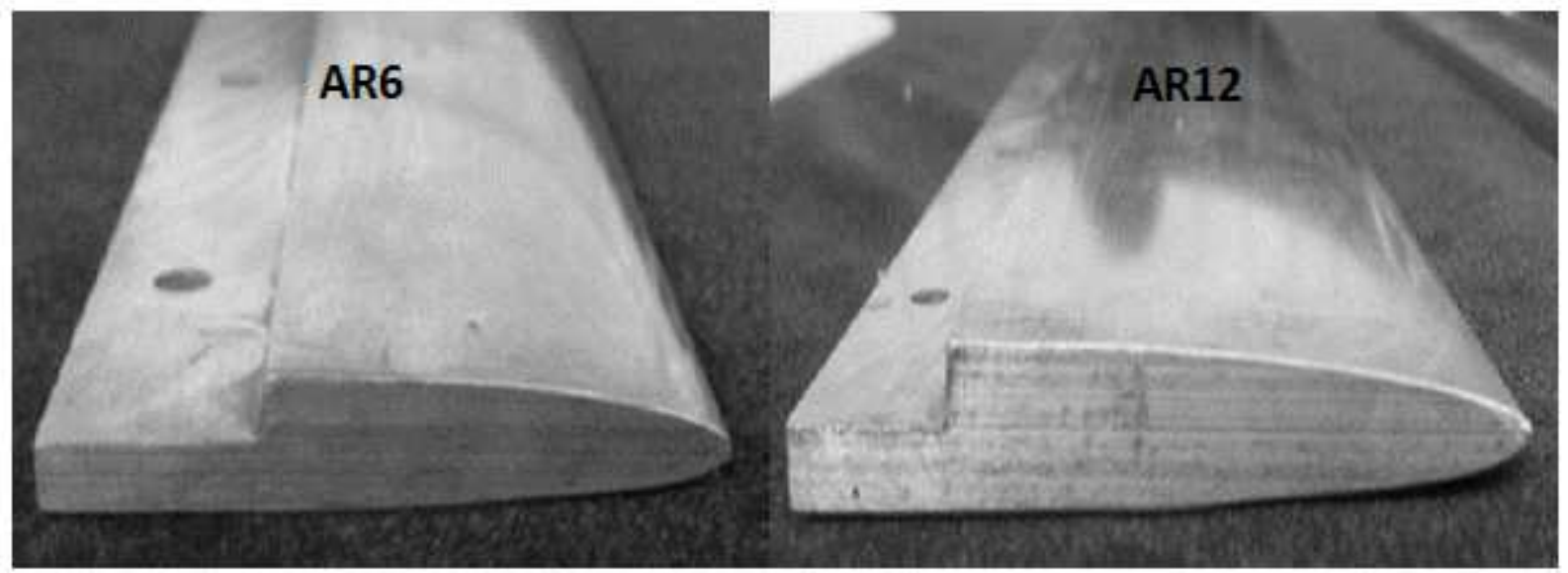




\section{Figure 3}

Sample leading edges for the wind tunnel test

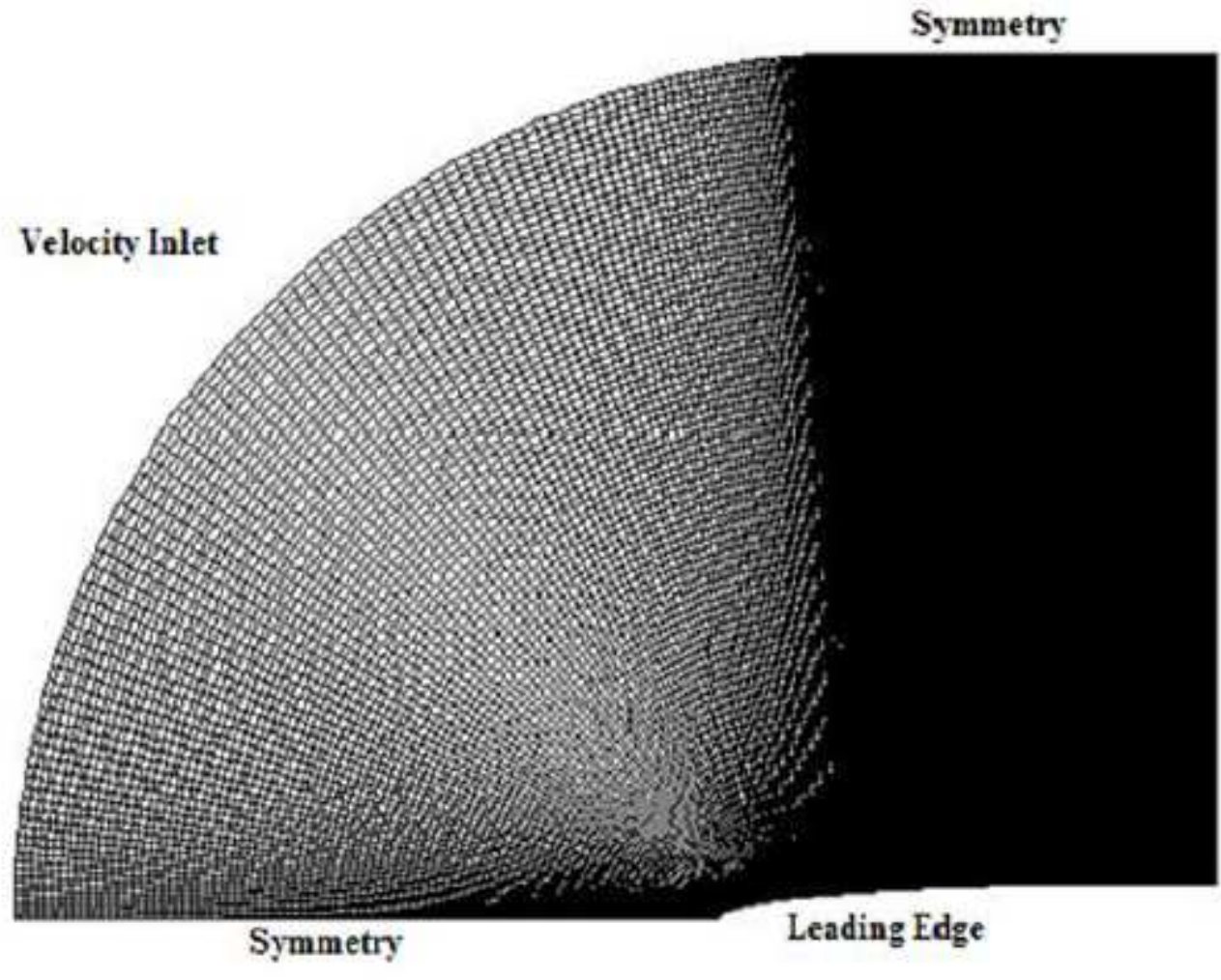

(a)

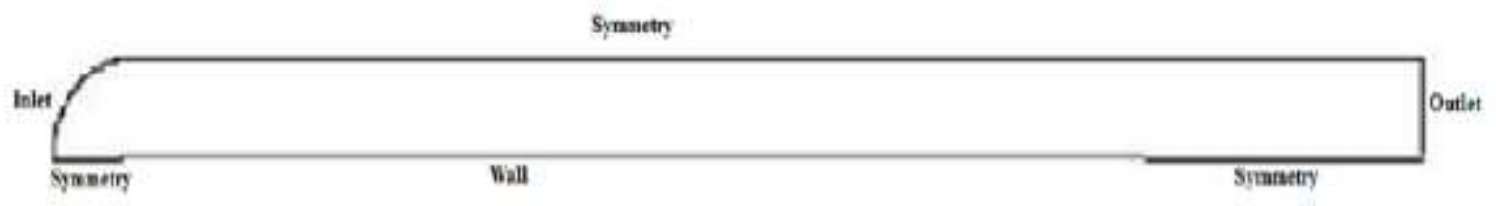

(b)

\section{Figure 4}

Mesh Distribution (a) and Boundary conditions (b) along the flat plate 


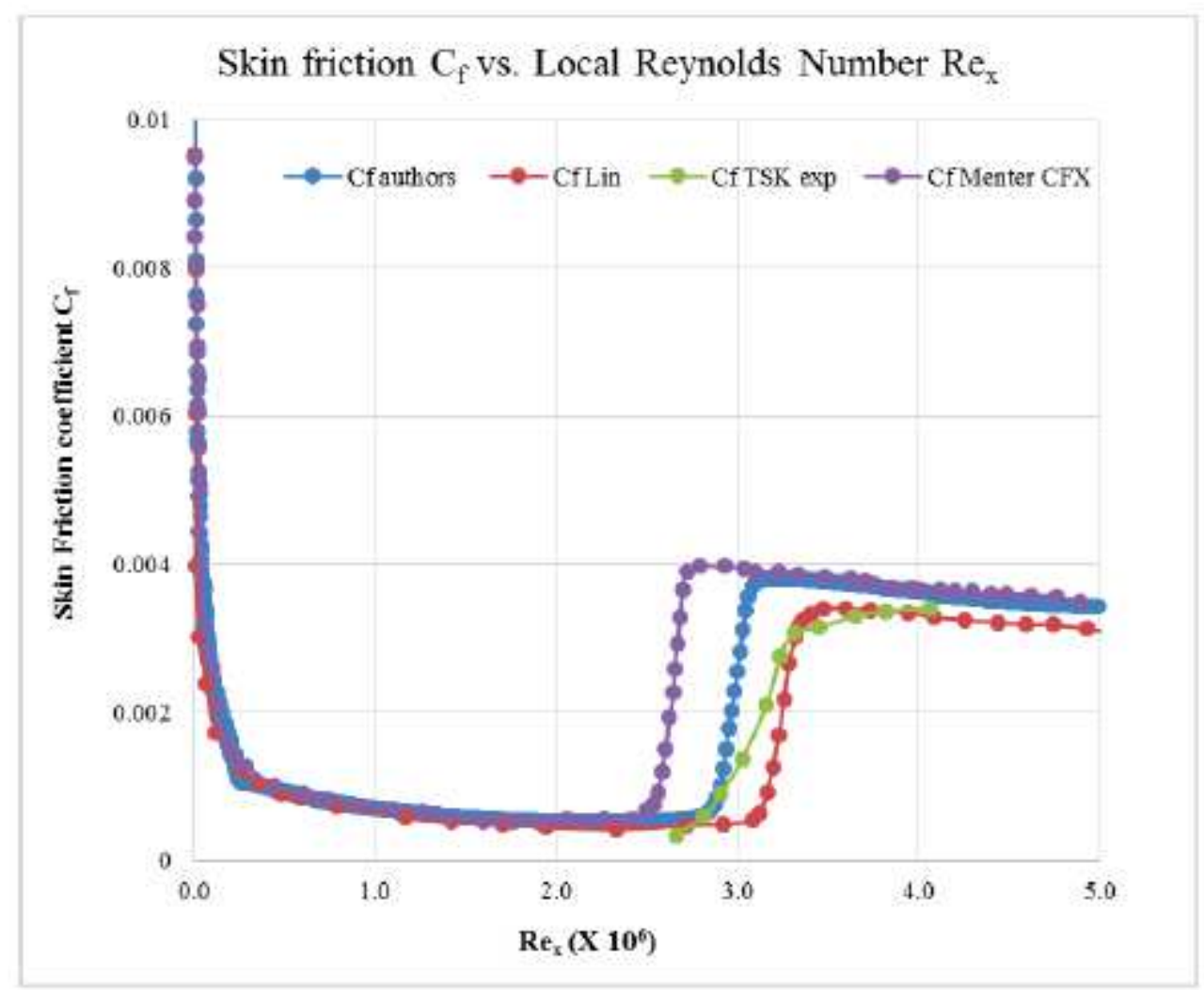

Figure 5

Benchmark graph of skin friction Cf vs. local Reynolds number Rex

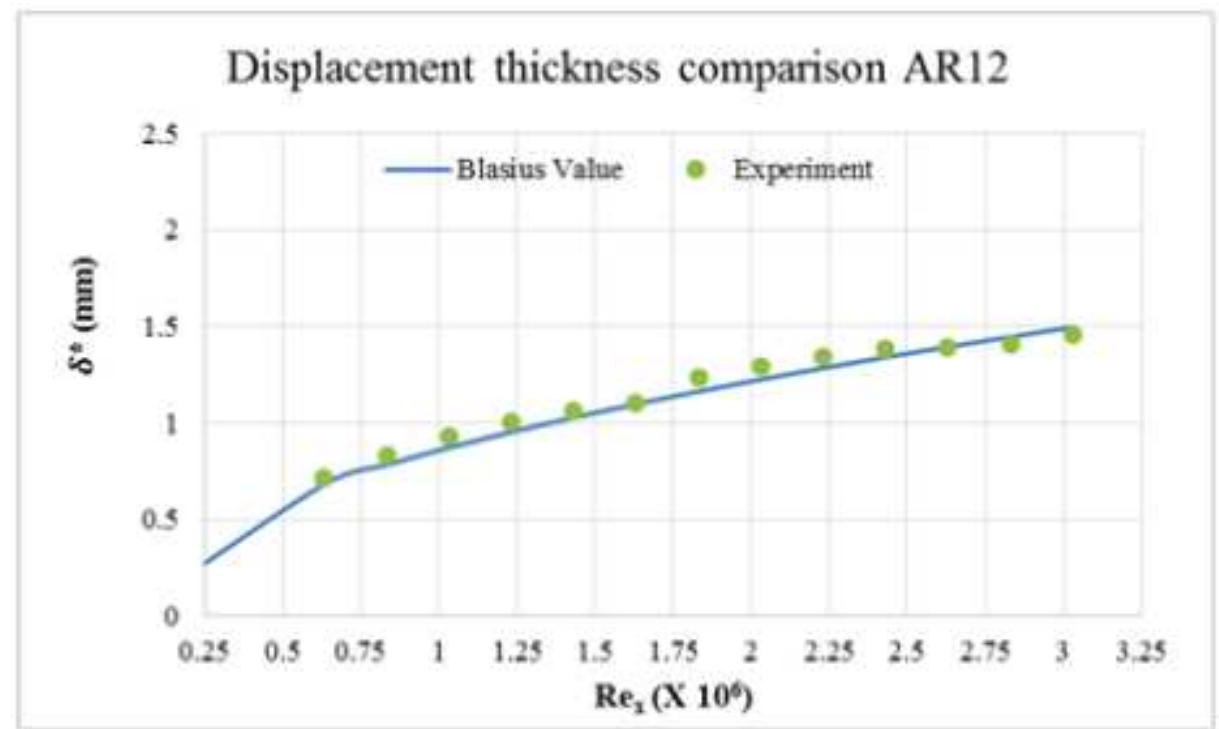

Figure 6

Displacement thicknesses comparison 


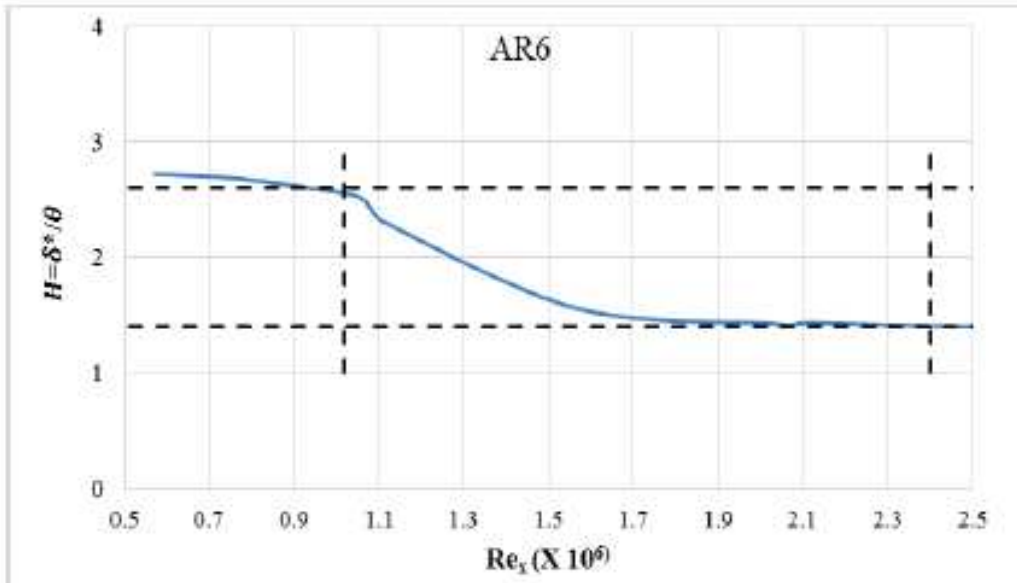

(a)

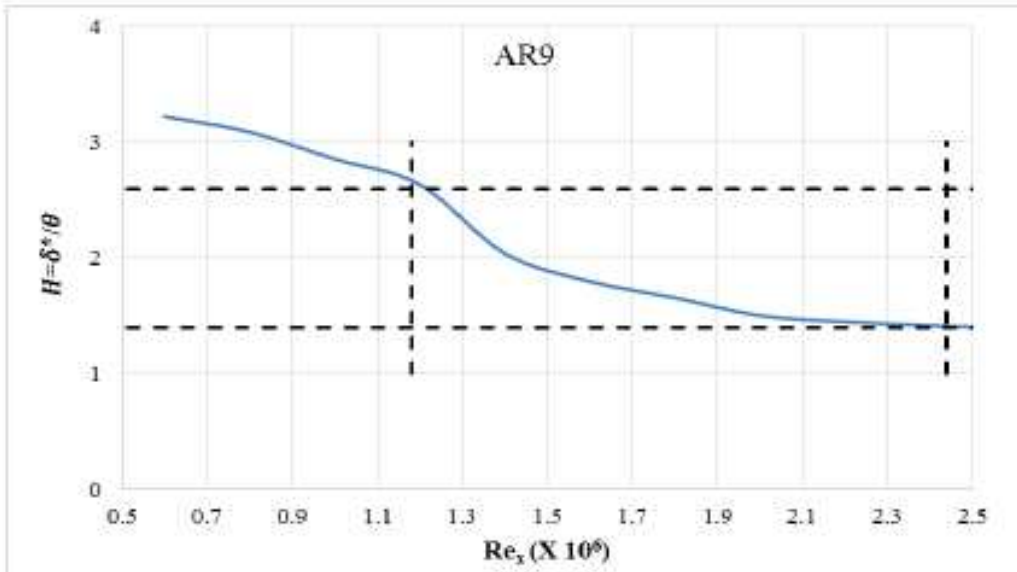

(b)

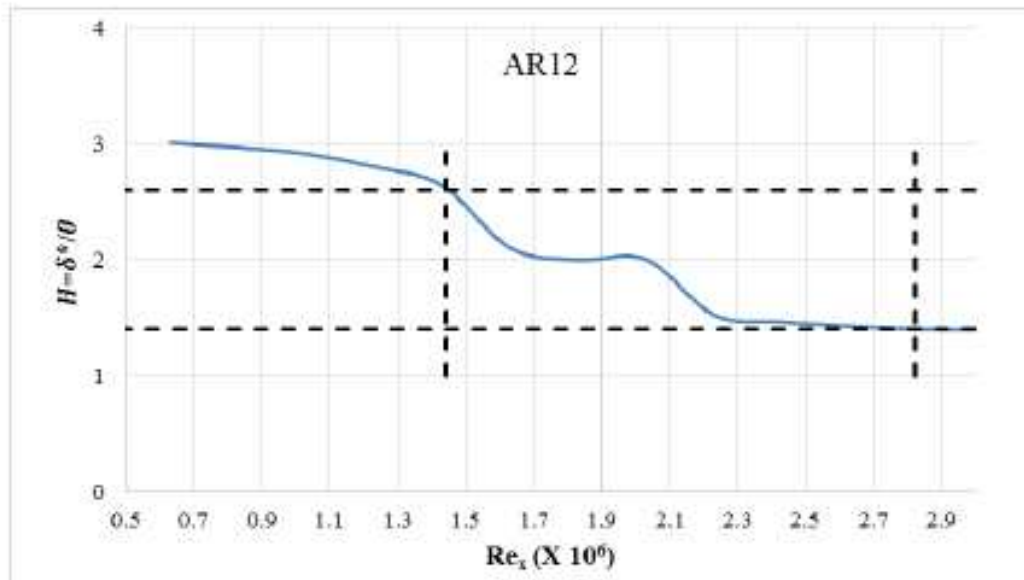

(c)

Figure 7

Shape factor $\mathrm{H}$ for different leading edges (Bhatia, et al., 2014) 


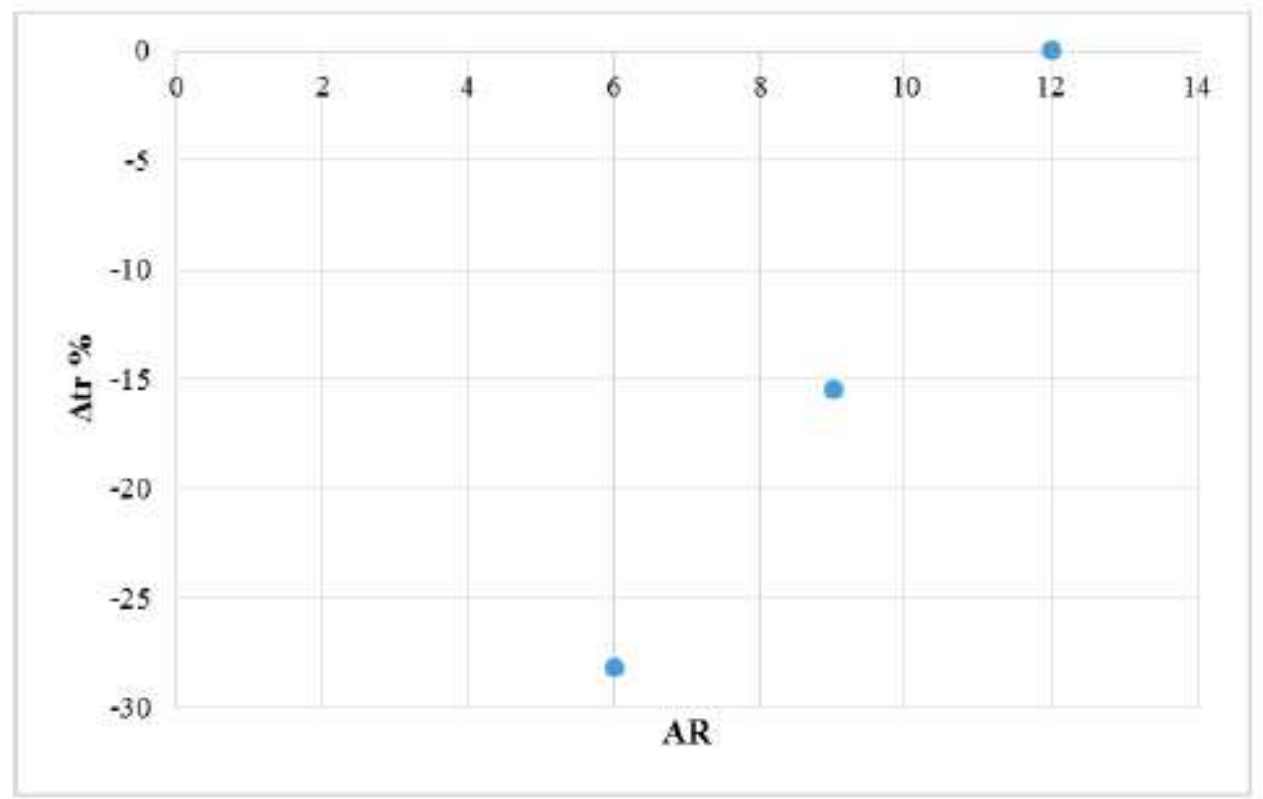

Figure 8

Experimental transition onset percentage difference between AR12, 9, 6

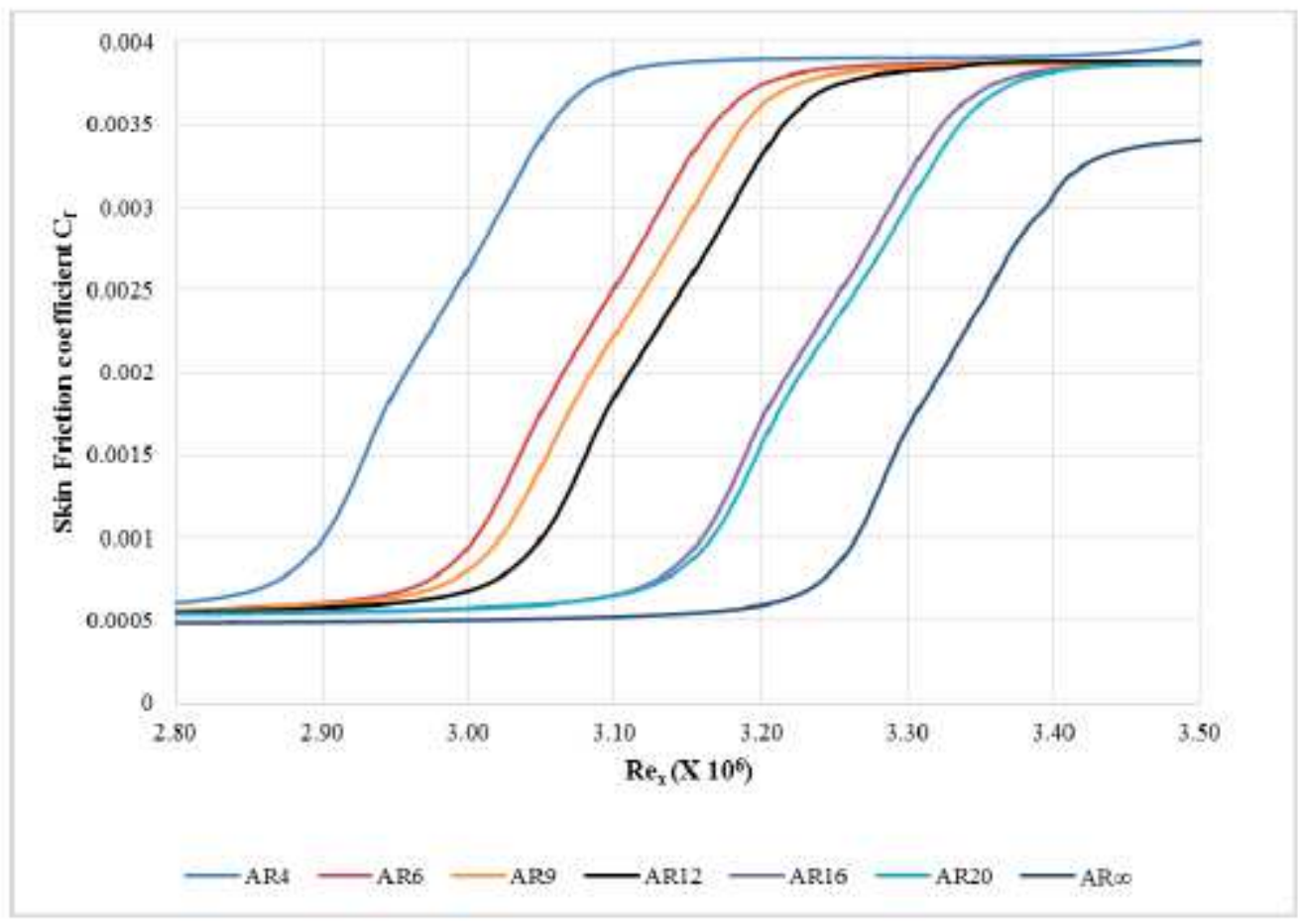

Figure 9

Cf values for AR4, 6,9,12, 16, 20 and $\infty$ 


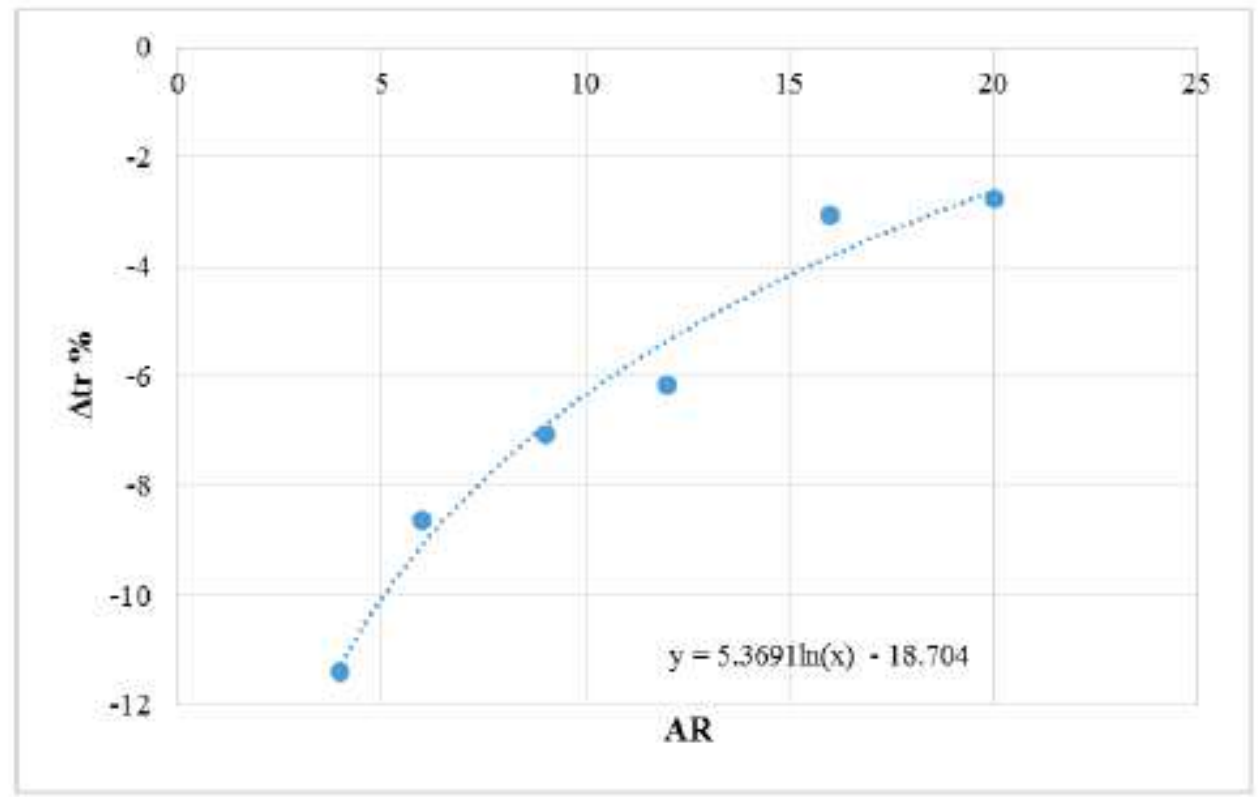

Figure 10

Transition onset percentage difference between $A R^{\infty}$ and other leading edges
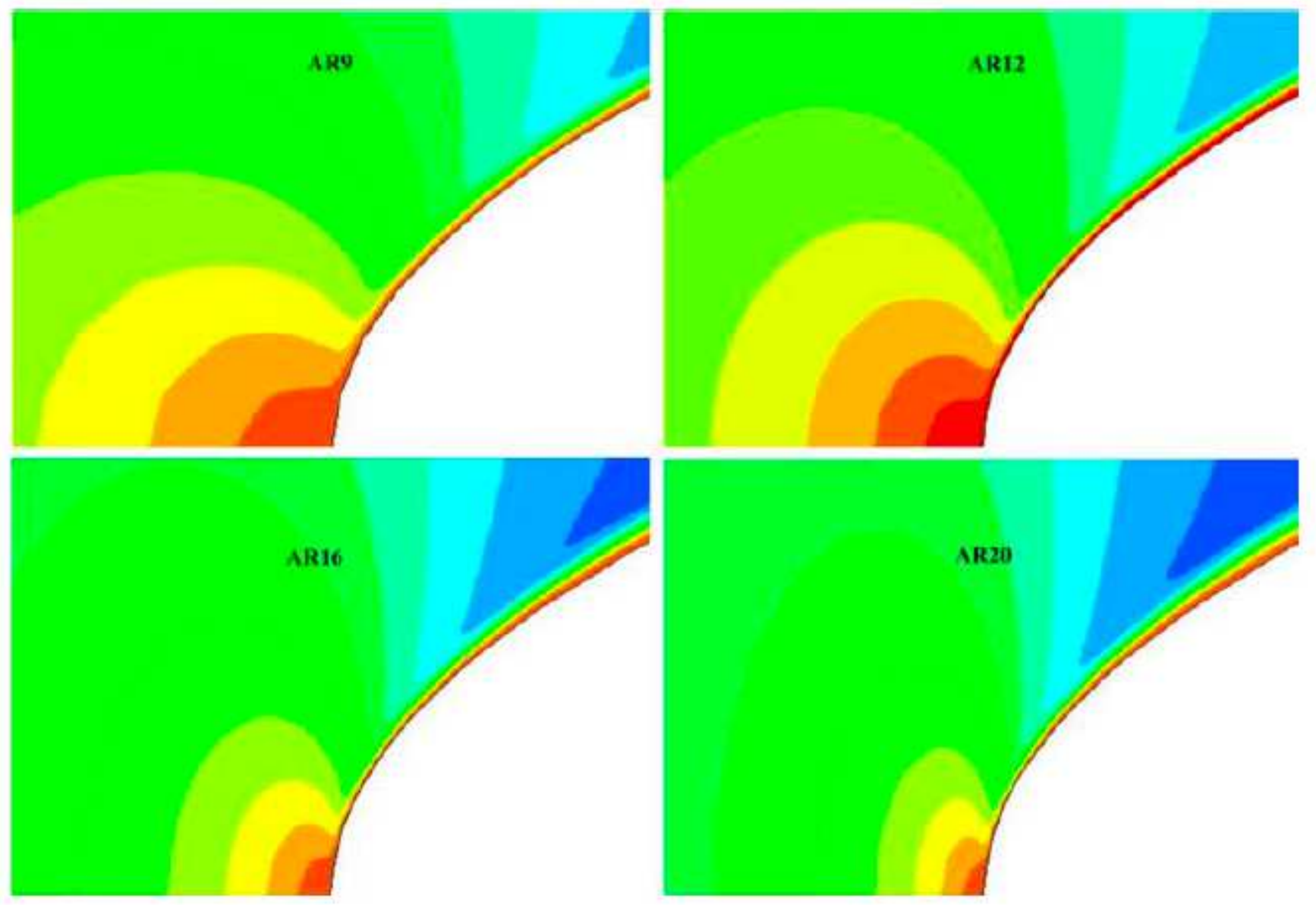

Figure 11 
Pressure contours at the stagnation region for different leading edges

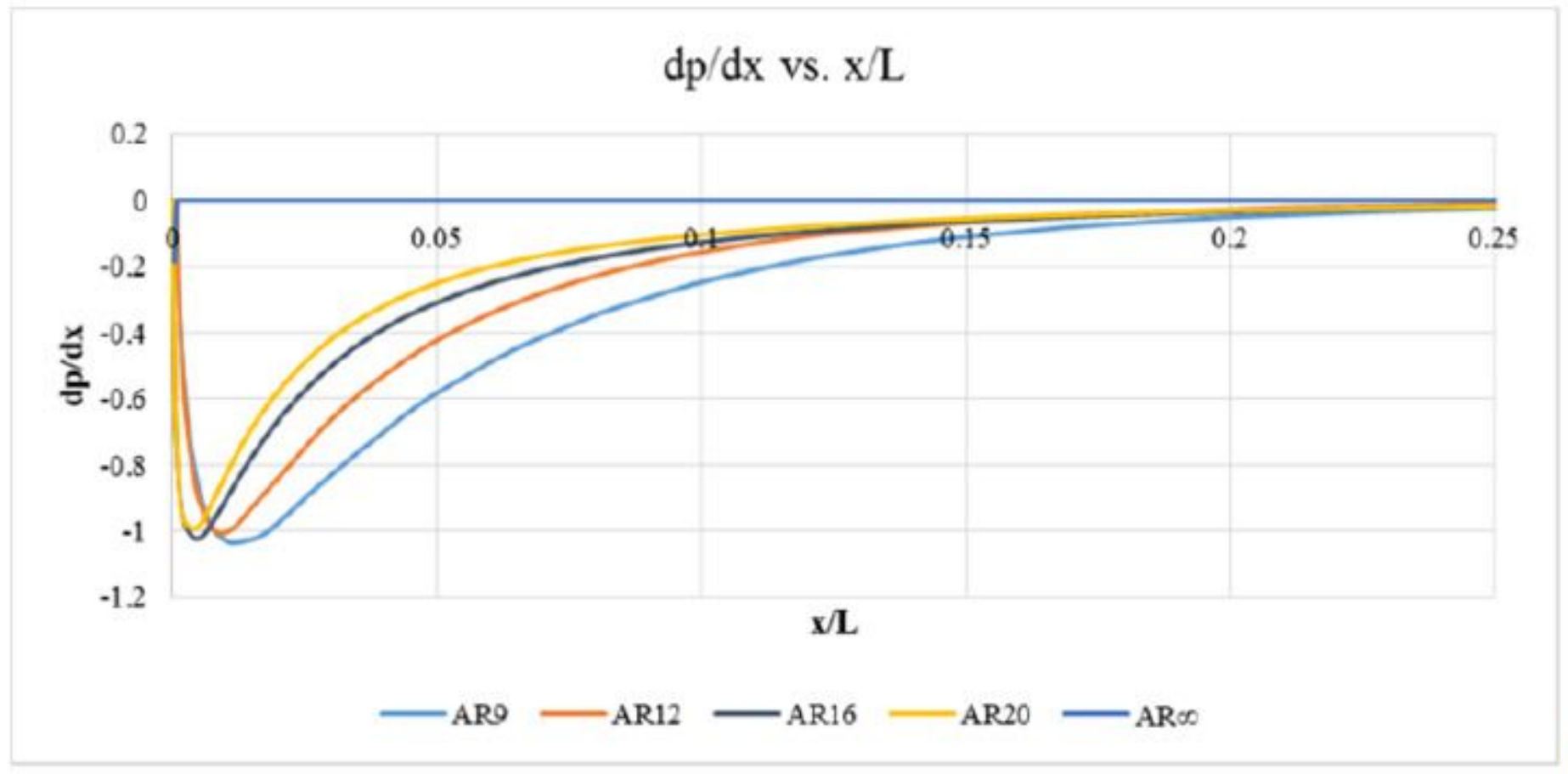

(a)

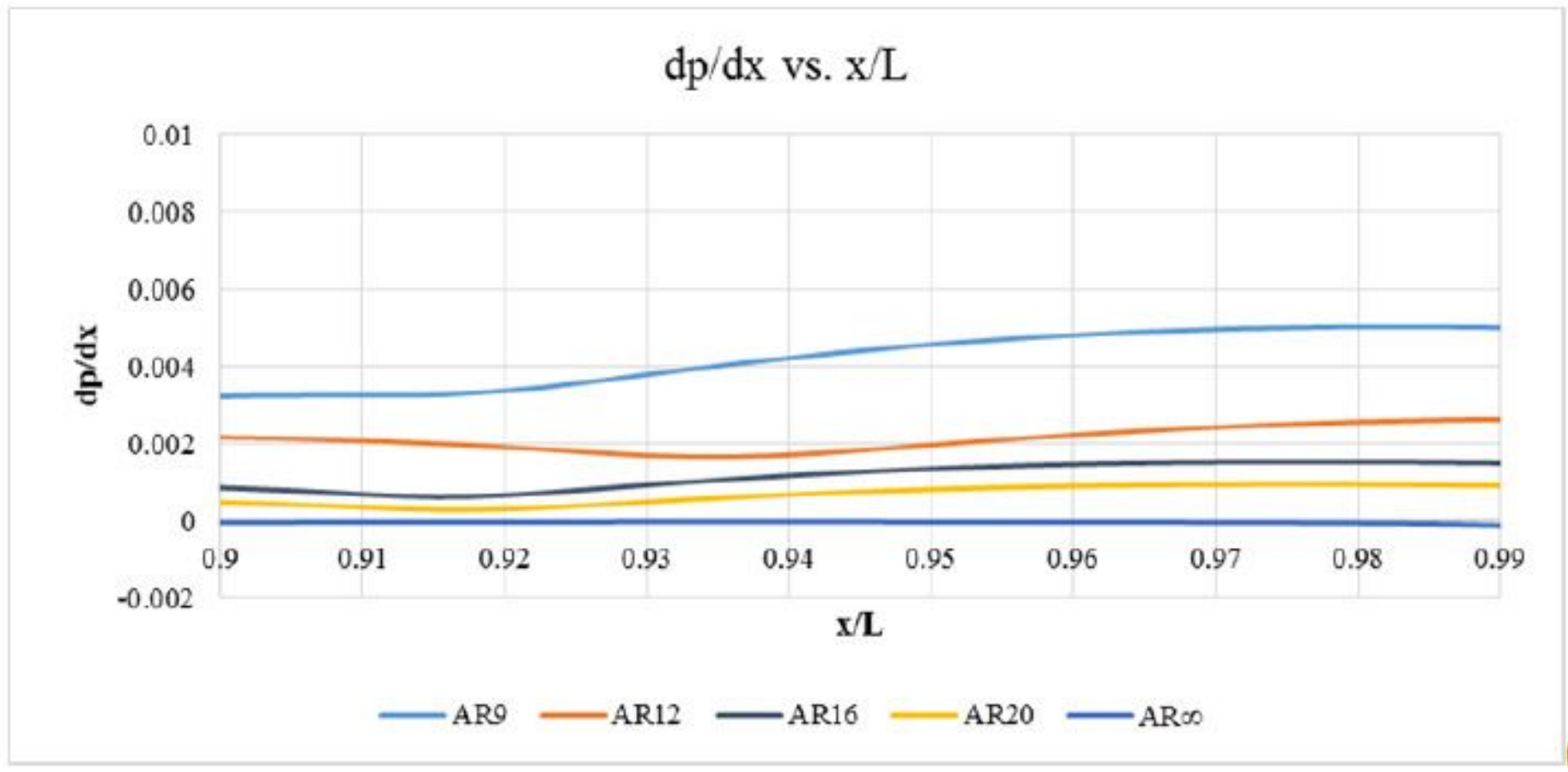

(b)

Figure 12

Pressure gradient for different leading edges 


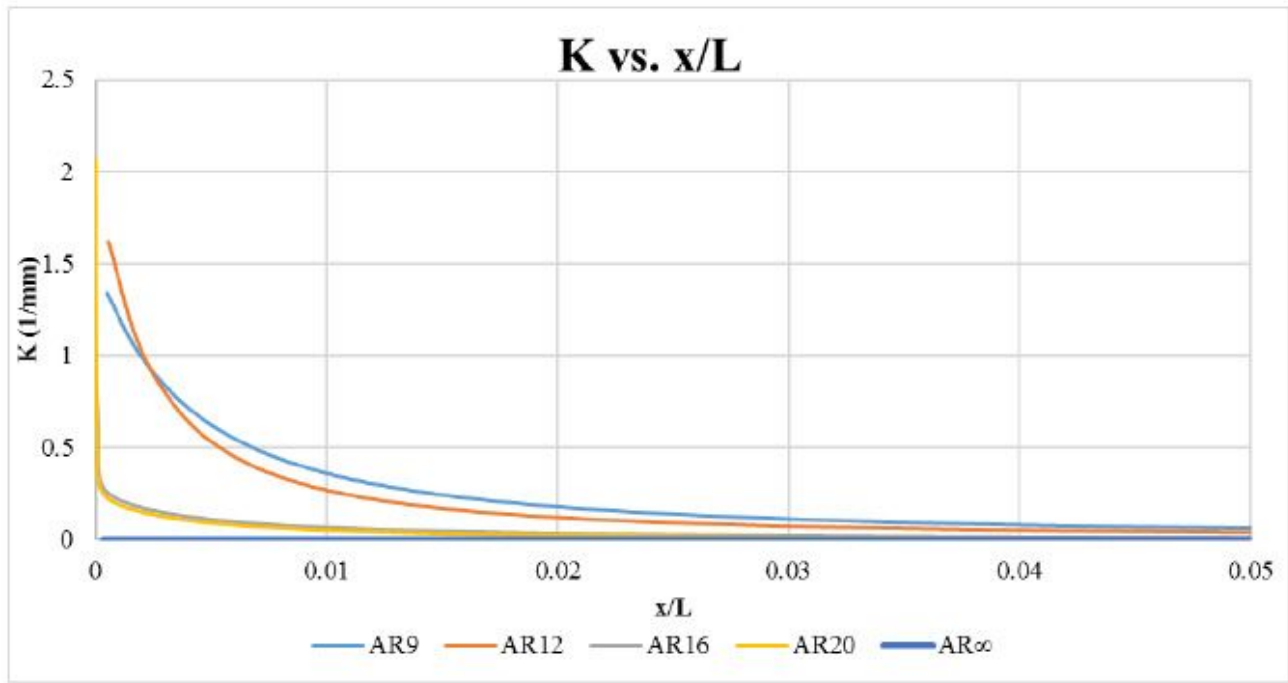

(a)

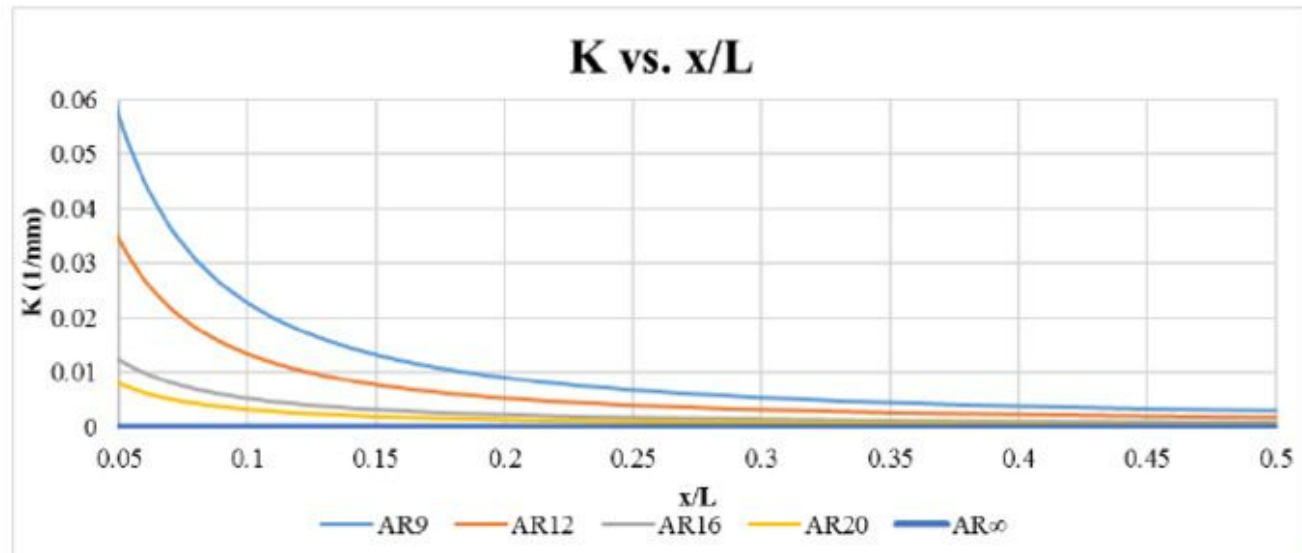

(b)

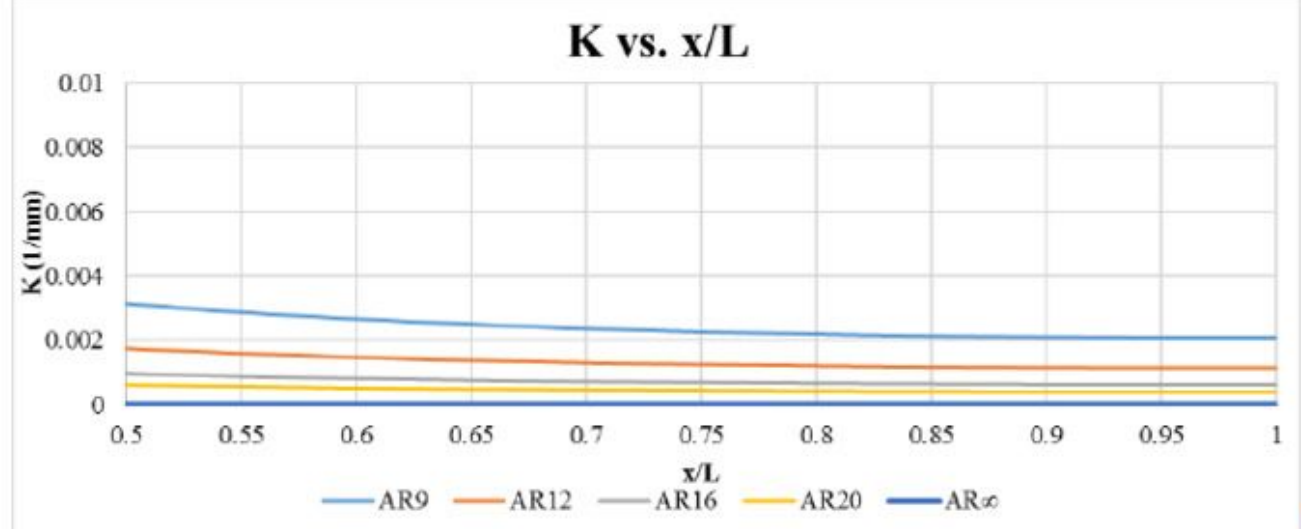

(c)

Figure 13

Curvature along the length of leading edge for different AR 


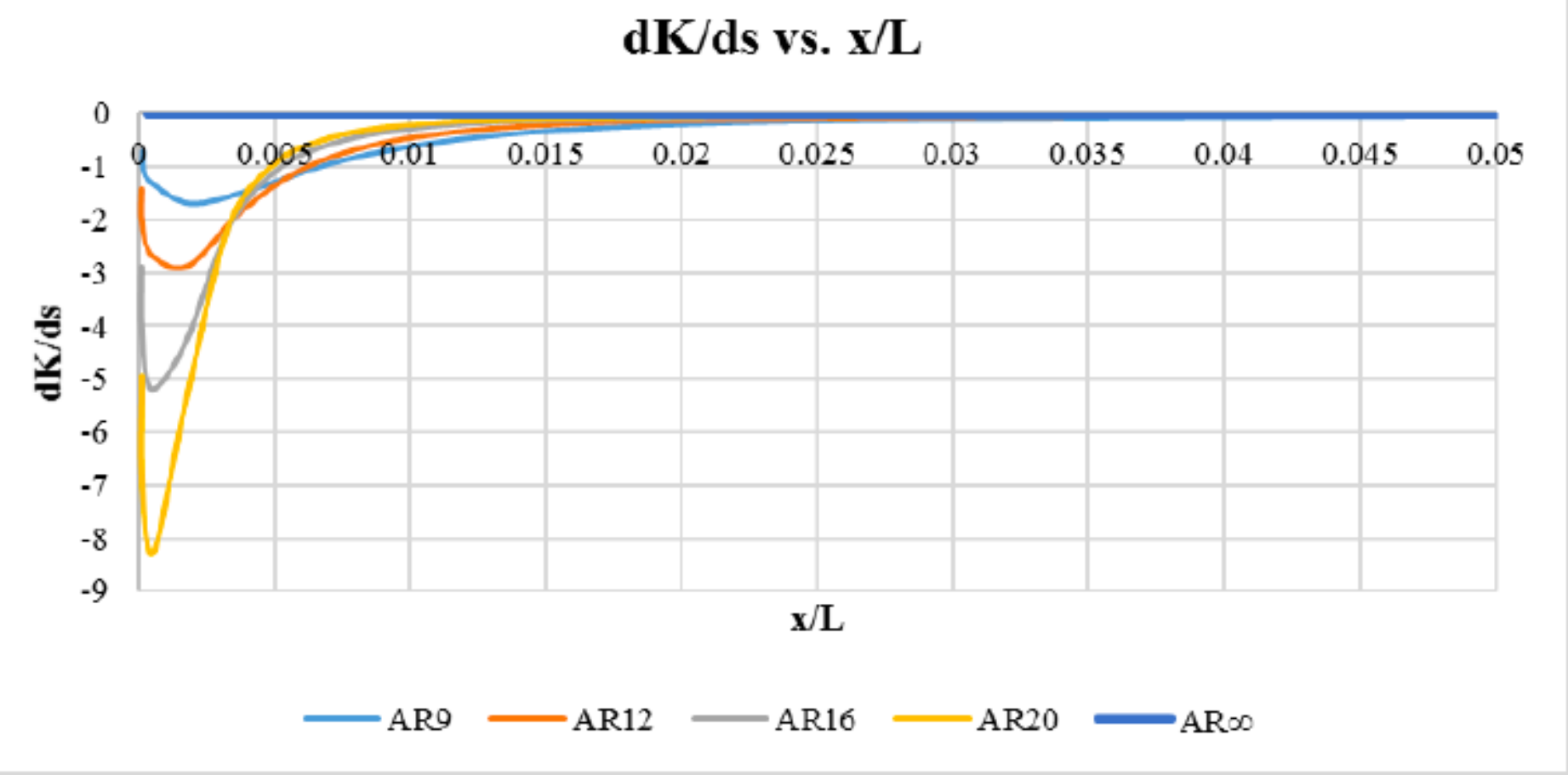

Figure 14

Curvature change along the length of leading edge for different AR

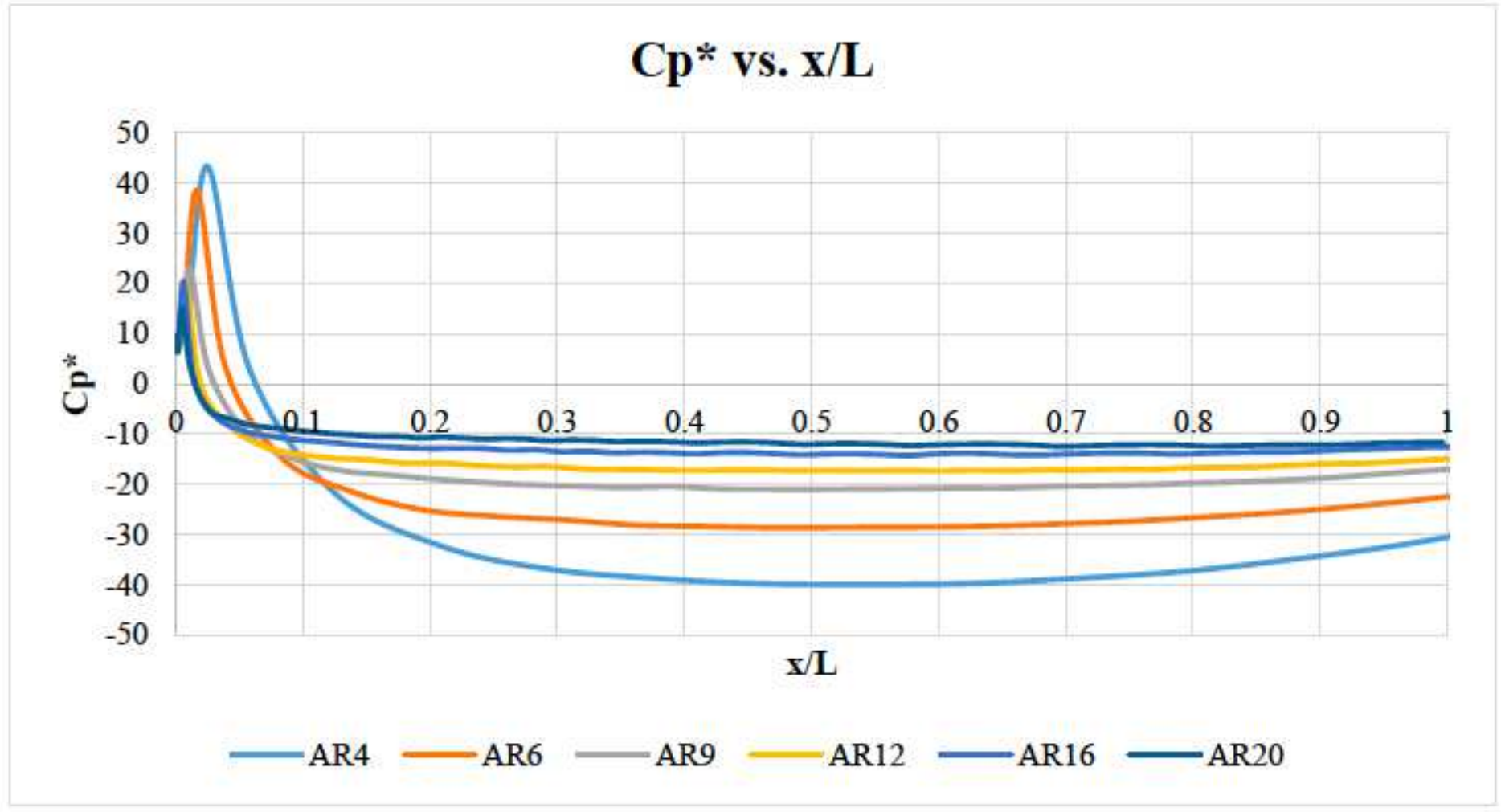

Figure 15 
Cp* vs. $x / L$ for different leading edges

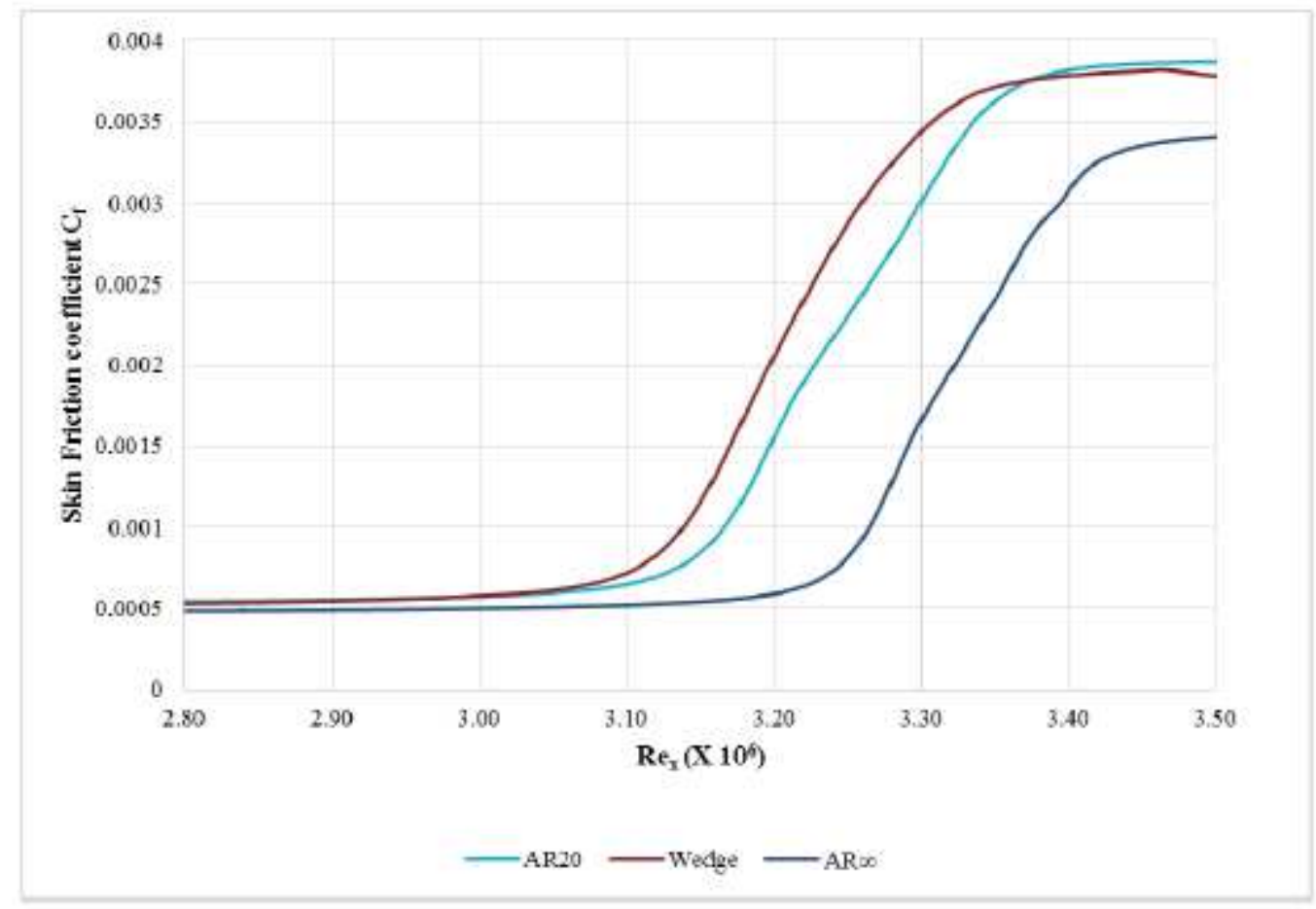

Figure 16

Skin friction coefficient for Wedge against AR20 and ARo

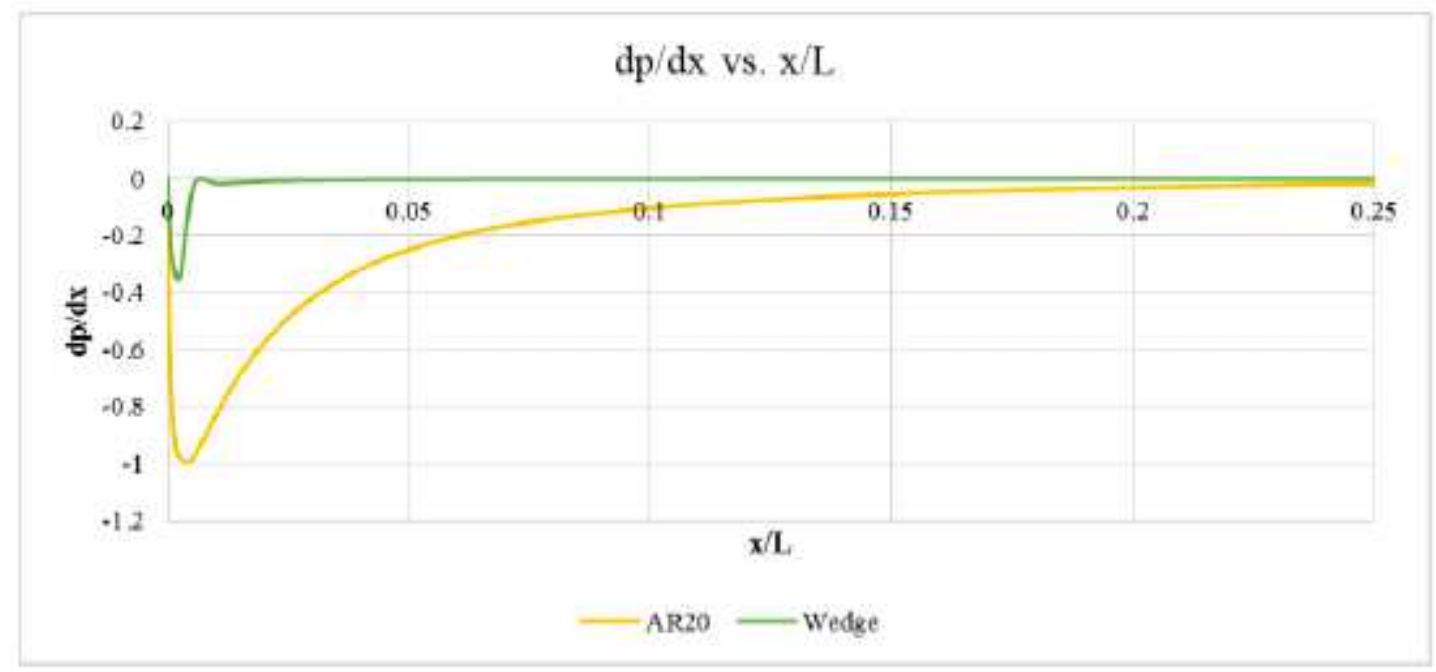

Figure 17

Pressure gradient for Wedge and AR20 


\section{Cp* vs. $\mathbf{x} / \mathbf{L}$}

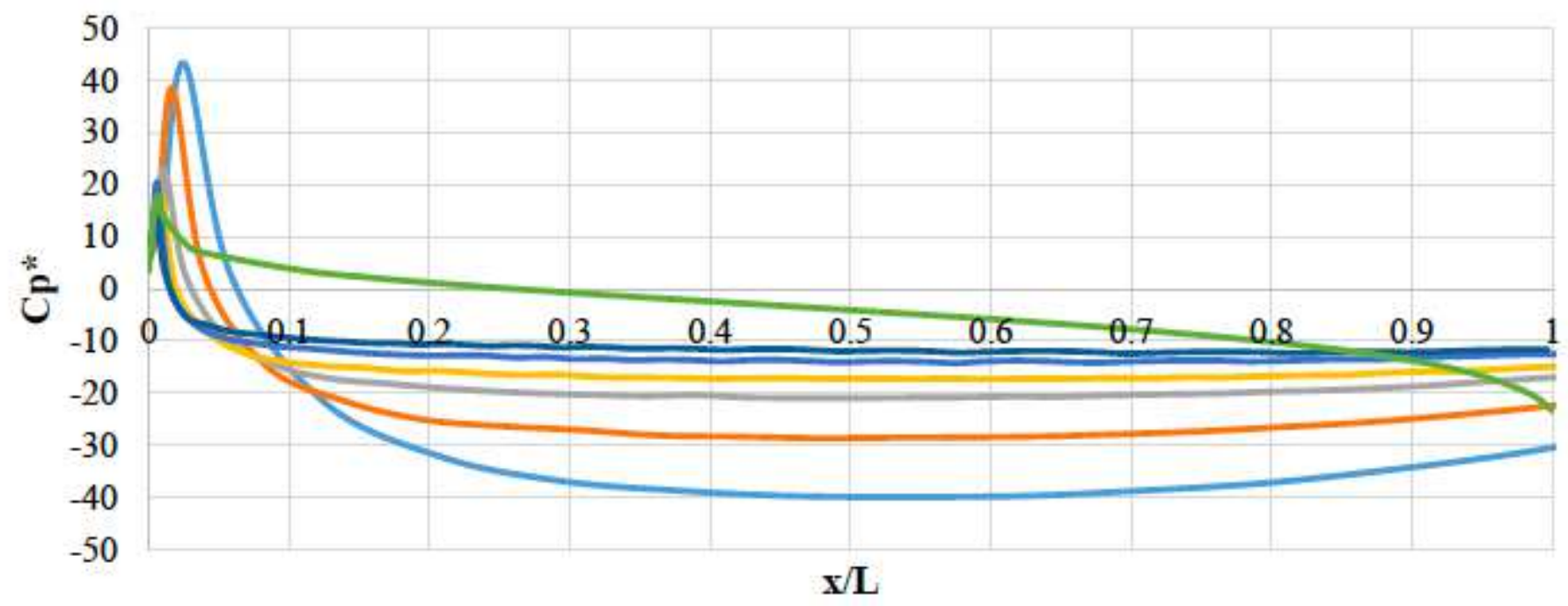

$-\mathrm{AR} 4-\mathrm{AR} 6-\mathrm{AR} 9-\mathrm{AR} 12-\mathrm{AR} 16-\mathrm{AR} 20-$ Wedge

Figure 18

Comparison of $\mathrm{Cp}{ }^{*}$ vs. $\mathrm{x} / \mathrm{L}$ for the wedge against different elliptical leading edges 